\title{
Study of the near-wall-turbulent region of the high-Reynolds-number boundary layer using an atmospheric flow
}

\author{
By GARY J. KUNKEL ${ }^{1}$ AND IVAN MARUSIC \\ ${ }^{1}$ Department of Mechanical and Aerospace Engineering, Princeton University, Princeton, \\ NJ 08544, USA \\ ${ }^{2}$ Department of Aerospace Engineering and Mechanics, University of Minnesota, Minneapolis, \\ MN 55455, USA
}

(Received 31 March 2005 and in revised form 29 July 2005)

Data from the near-wall-turbulent region of the high-Reynolds-number atmospheric surface layer are used to analyse the attached-eddy model of wall turbulence. All data were acquired during near-neutral conditions at the Surface Layer Turbulence and Environmental Science Test (SLTEST) facility located in the western Utah Great Salt Lake Desert. Instantaneous streamwise and wall-normal components of velocity were collected with a wall-normal array of two-component hot wires within the first $2 \mathrm{~m}$ above the surface of the salt flats. Streamwise and wall-normal turbulence intensities and spectra are directly compared to corresponding laboratory data and similarity formulations hypothesized from the attached-eddy model of wall turbulence. This affords the opportunity to compare results with Reynolds numbers varying over three orders of magnitude. The wall-normal turbulence-intensity similarity formulation is extended. The results show good support for the similarity arguments forwarded by the attached-eddy model as well as Townsend's (1956) Reynolds-number similarity hypothesis and lack of the 'inactive' motion influence on the wall-normal velocity component. The effects of wall roughness and the spread in the convection velocity due to this roughness are also discussed.

\section{Introduction}

An experimental investigation of the near-wall-turbulent (logarithmic) region of the high-Reynolds-number turbulent boundary layer was conducted. The main purpose of this work is to use these data to investigate the attached-eddy model of wallbounded turbulence at high Reynolds numbers. (A good review of the importance of understanding high-Reynolds-number wall-bounded flow is given by Gad-el-Hak \& Bandyopadhyay 1994.) The attached-eddy model essentially provides a kinematic description for wall-bounded turbulence. The foundation of the model, which is based on the attached-eddy hypothesis of Townsend (1976), is the observation that wallbounded turbulence contains a collection of coherent structures or eddies. Therefore, the model proposes that the statistical features of wall-bounded turbulence can be modelled by a linear superposition of such eddies. The model has been refined and developed over the past three decades on the data of low-to-moderate Reynoldsnumber wall-bounded turbulence experiments (e.g. pipe and boundary-layer flow) and has led to a number of similarity laws. For a complete review of the attached-eddy model see Perry \& Marusic (1995) and the references therein. 
One of the strengths of the attached-eddy model is the physical framework it provides for the analysis and understanding of the mechanism of wall-bounded turbulence. Perry \& Abell (1975), Perry, Henbest \& Chong (1986), and Perry \& $\mathrm{Li}$ (1990) used this framework along with simple dimensional-analysis arguments applied to the velocity contributions of the attached eddies to suggest several regions of spectral scaling. They further integrated the spectra over these different scaling regions to obtain turbulence-intensity similarity formulations. These turbulenceintensity similarity formulations, and the spectral similarity arguments on which they are based, have been tested for a variety of wall-bounded flows and the results used to further improve the model. For instance, the suggested formulations have been investigated using smooth-pipe flow (Perry et al. 1986), smooth and rough zero-pressure-gradient turbulent boundary layers (Perry \& Li 1990), and smooth favourable- and adverse-pressure-gradient turbulent boundary layers (Jones, Marusic \& Perry 2001 and Marusic \& Perry 1995, respectively). The streamwise formulation has also been compared with experimental data over a wide range of Reynolds numbers and extended in the works by Marusic et al. (1997) and Marusic \& Kunkel (2003) (the original formulations were only valid in the log region). While the different similarity arguments and statistics hypothesized by the attached-eddy model have been tested with data over a large range of Kármán numbers $\delta^{+}\left(\equiv \delta U_{\tau} / v\right) \sim 10^{3}-10^{4}$, they have not been compared to data at high Kármán numbers $\left(\delta^{+} \sim 10^{6}\right)$, which corresponds with many practical applications. Here, $\delta$ is the boundary-layer thickness, $U_{\tau}$ is the mean wall shear velocity, and $v$ is the kinematic viscosity. Also, " ${ }^{+}$' represents inner-flow scaling (i.e. $U_{\tau}$ or $v / U_{\tau}$ ). The focus of this study is to analyse the turbulenceintensity similarity formulations, and the spectral similarity arguments on which they are based, at high Reynolds numbers. As observed by Raupach, Antonia \& Rajagopalan (1991), an evaluation of the similarity formulations is a critique of the model itself and therefore analysing the high-Reynolds-number turbulence intensities and spectra can provide an experimental verification of the underlying principles of the model.

There are different ways of obtaining high-Reynolds-number flows and each way has its own challenges. Two common laboratory methods are increasing the length scale of the flow (e.g. Saddoughi \& Veeravalli 1994; Fernholz et al. 1995) and increasing the density of the flow (e.g. DeGraaff \& Eaton 2000; Morrison et al. 2004) achieving Kármán numbers $\sim 10^{5}, 10^{4}, 10^{5}$ and $10^{5}$, respectively. However, large facilities are costly to run and increasing the density necessarily means decreasing the smallest scales in the flow (keeping a fixed outer-scale) requiring instrumentation with a resolution smaller than what currently exists. We avoid both of these problems, and obtain a higher Reynolds number, by using the exceptionally large length-scale atmospheric boundary layer (ABL). Experimenting in the ABL also has its challenges such as the unpredictability of the weather, the non-uniform wall conditions owing to vegetation or hills, the convective flow from the wall owing to the diurnal heating of the sun, and accessibility of measuring equipment. However, using a well-chosen site such as the Surface Layer Turbulence and Environmental Science Test (SLTEST) facility in the western Utah salt flats, and acquiring data when the boundary layer is near neutral stability can reduce many of these challenges. A more detailed discussion of this specific site and its use is given in $\S 3$.

Perhaps the most significant reservation of using the atmosphere to mimic a canonical high-Reynolds-number wall-bounded flow that cannot be remedied by taking data at appropriate times, or choosing an appropriate site, is that the outer 
boundary condition and large-scale $(\sim \mathrm{km})$ structure of the atmospheric boundary layer is different from that in a canonical flat-plate boundary layer. The atmospheric boundary layer is bounded on its upper surface by the free atmosphere with near geostrophic winds and sporadic turbulence in convective clouds or near jet streams (Stull 1988). Furthermore, the ABL is made of up several physically different layers. Historically, it has been described as containing an upper mixed layer, $\sim 1 \mathrm{~km}$, where the turbulence is typically convectively driven and a lower atmospheric surface layer (ASL), $\sim 100 \mathrm{~m}$, where the turbulence in near-neutrally stable flows is mechanically shear driven. Hunt \& Carlotti (2001) and Drobinski et al. (2004) have further decomposed the ASL into the upper shear surface layer (SSL), $\sim 100 \mathrm{~m}$, and the near-wall eddy surface layer $(\mathrm{ESL}), \sim 10 \mathrm{~m}$. The dominating mechanism in the SSL is the shearing of isotropic turbulence while in the ESL it is the blocking of the eddies owing to the wall.

For a more in-depth review of the structure of the ABL, see Stull (1988), Hogstrom, Hunt \& Smedman (2002), and Drobinski et al. (2004). It is fairly clear that the large-scale characteristics of the typical ABL poorly represent the canonical turbulent boundary layer. However, here we limit our study to the first $2 \mathrm{~m}$ above the wall (the lower $20 \%$ of the ESL), and conduct our experiments at a unique site, with extremely flat and uniform terrain. Under neutrally stable conditions we therefore expect to have captured the essential features of a high-Reynolds-number wall-bounded flow dominated by shear and blocking similar to a canonical turbulent boundary layer (as suggested by Hunt \& Carlotti 2001). While it is evident that higher in the surface layer the structure of turbulence is different, these differences are of secondary importance this close to the wall, and this is the region of most interest for our investigation.

The main experimental aim of this study was to acquire high-Reynolds-number data in the $\log$ region of the flow where the wall-normal position relative to the boundary-layer thickness is very small. That is, have $z / \delta \rightarrow 0$ while $z^{+} \gtrsim 100$. The SLTEST facility is ideal in allowing us to achieve this. Furthermore, the relatively large length and time scales involved ensure that small-scale spatial and temporal resolution is achieved with conventional hot-wire anemometry.

Along with an evaluation of the specific similarity formulations derived from the attached-eddy model, the high-Reynolds-number atmospheric-surface-layer data are also used to consider the effects of roughness on the turbulence intensities and thereby test the Townsend (1956) Reynolds-number similarity hypothesis. This is another advantage specific to the SLTEST facility. The site gives us a high-Reynoldsnumber rough-wall turbulent boundary layer with a very small roughness height with respect to the boundary-layer thickness. In light of the data, an explanation is proposed for the recent discrepancy between those that find outer-flow similarity (e.g. Flack, Schultz \& Shapiro 2005) and those who do not (e.g. Krogstad \& Antonia 1999; Bhaganagar, Kim \& Coleman 2004). Consistent with Perry \& Li (1990) we also find the streamwise spectra must be interpreted cautiously because of the use of Taylor's hypothesis and the spread in convection velocities in rough-wall turbulent boundary layers. This will be discussed further in $\S 4.3$.

The extended similarity formulations are reviewed in $\S 2$. Section 3 describes the SLTEST facility and discusses some the subtleties of testing in the atmosphere. Section 4 gives the results of the atmospheric study and compares them to the similarity formulations and expected spectral similarity regions. For comparison, laboratory data from appropriate sources are always compared to this atmospheric high-Reynolds-number data. It is crucial to keep in mind that while the atmospheric 
data by nature contain a higher uncertainty (discussed in $\S 3.4$ ), the Reynolds number is two to three orders of magnitude larger than similar laboratory studies and therefore any Reynolds number effects are more easily deducible.

\section{Turbulence-intensity similarity formulations}

Throughout this paper $u_{1}$ and $u_{3}$ refer to the fluctuating velocities in the streamwise $(x)$ and wall-normal $(z)$ directions, respectively.

\subsection{Streamwise component}

Marusic \& Kunkel (2003) extended the streamwise turbulence-intensity similarity formulation refined by Marusic et al. (1997) to be applicable across the entire smoothwall zero-pressure-gradient turbulent boundary layer. The streamwise turbulenceintensity similarity formulation is

$$
\overline{u_{1}^{2}}= \begin{cases}f_{I 1}\left[z^{+}\right] f_{T 1}\left[z^{+}, \delta^{+}\right] & \text {for } z^{+} \leqslant z_{\text {inner }}^{+} \\ f_{O 1}\left[z^{+}, \delta^{+}\right] & \text {for } z^{+} \geqslant z_{\text {outer }}^{+} \\ \text {cubic blend } & \text { for } z_{\text {inner }}^{+}<z^{+}<z_{\text {outer }}^{+}\end{cases}
$$

where

$$
\begin{gathered}
f_{I 1}=\frac{0.16\left(z^{+}\right)^{2}}{\left(1+a_{1}\left(z^{+}\right)^{2}\right)^{1 / 2}\left(1+\left(a_{2} z^{+}\right)^{2 a_{3}}\right)^{1 / 2}}, \\
f_{T 1}=1+(\alpha-1) \frac{\ln \left[z^{+}\right]}{\ln \left[\left(z^{+}\right)_{r e f}\right]},
\end{gathered}
$$

and

$$
f_{O 1}=B_{1}-A_{1} \ln \left[\frac{z}{\delta}\right]-V_{g 1}\left[z^{+}, \frac{z}{\delta}\right]-W_{g 1}\left[\frac{z}{\delta}\right] .
$$

The inner part of the formulation $\left(f_{I 1}\right)$ is an empirical curve fit of high-resolution experimental data and the outer part $\left(f_{O 1}\right)$ is the Marusic et al. (1997) formulation. $f_{I 1}$ is scaled with $f_{T 1}$ such that the outer part of $f_{I 1}$ increases like the inner part of $f_{O 1}$. Here,

$$
\alpha=\frac{B_{1}-A_{1} \ln \left[\left(z^{+}\right)_{r e f} / \delta^{+}\right]}{B_{1}-A_{1} \ln \left[\left(z^{+}\right)_{r e f} /\left(\delta^{+}\right)_{r e f}\right]},
$$

is the value of $f_{T 1}$ at $\left(z^{+}\right)_{\text {ref }}=50$ obtained from the asymptotic form of $f_{O 1}$. As before, $A_{1}=1.03, B_{1}=2.39, z_{\text {inner }}^{+}=30, z_{\text {outer }}^{+}=150, a_{1}=0.008, a_{2}=0.115$ and $a_{3}=1.6$. From Marusic et al. (1997),

$$
V_{g 1}\left[z^{+}, \frac{z}{\delta}\right]=S_{A}\left[z^{+}\right]\left(E_{1}-A_{1} \ln \left[z^{+}\right]\right)+S_{V}\left[z^{+}\right] V\left[z^{+}\right]\left(1-\frac{z}{\delta}\right)
$$

is the viscous deviation from the log-region high-Reynolds-number asymptotic form

$$
{\overline{u_{1}^{2}}}^{+}=B_{1}-A_{1} \ln \left[\frac{z}{\delta}\right]
$$

and the wake deviation is given by

$$
W_{g 1}\left[\frac{z}{\delta}\right]=B_{1}\left(\frac{z}{\delta}\right)^{2}\left(3-2 \frac{z}{\delta}\right)-A_{1}\left(\frac{z}{\delta}\right)^{2}\left(1-\frac{z}{\delta}\right)\left(1-2 \frac{z}{\delta}\right) .
$$


The formulation is based on the idea that the attached-eddy motions in the log region and beyond impose a forcing on the viscous buffer zone and sublayer. This outer layer forcing yields the Reynolds-number dependence in the inner portion of the layer. Marusic \& Kunkel (2003) found the extended streamwise formulation to describe data well, over a Kármán number range $10^{3}-10^{6}$. Based on the success of the streamwise formulation, the wall-normal formulation is also extended to be applicable across the entire smooth-wall zero-pressure-gradient turbulent boundary layer.

\subsection{Wall-normal component}

Using similar arguments based on the attached-eddy hypothesis the wall-normal formulation is expected to follow

$$
{\overline{u_{3}^{2}}}^{+}= \begin{cases}f_{13}\left[z^{+}\right] & \text {for } z^{+} \leqslant z_{\text {inner }}^{+} \\ f_{O 3}\left[z^{+}, \delta^{+}\right] & \text {for } z^{+} \geqslant z_{\text {outer }}^{+} \\ \text {cubic blend } & \text { for } z_{\text {inner }}^{+}<z^{+}<z_{\text {outer }}^{+}\end{cases}
$$

As with the streamwise formulation, we chose $z_{\text {inner }}^{+}=30$ and $z_{\text {outer }}^{+}=150$. The exact values are of secondary importance. Again, the inner portion is obtained from an empirical curve fit of high-resolution experimental data,

$$
f_{I 3}\left[z^{+}\right]=c_{1} z^{+4}\left[\left(1+c_{2} z^{+4}\right)\left(1+c_{3} z^{+4}\right)\left(1+c_{4} z^{+4}\right)\left(1+c_{5} z^{+4}\right)\right]^{-1 / 4},
$$

where $c_{1}=6.09 \times 10^{-4}, c_{2}=2.50 \times 10^{1}, c_{3}=1.62 \times 10^{-5}, c_{4}=4.62 \times 10^{-4}$, and $c_{5}=$ $1.96 \times 10^{-7}$. The function was chosen so as to have the correct near-wall behaviour as $z^{+} \rightarrow 0$, namely $f_{I 3} \rightarrow\left(c z^{+2}\right)^{2}$, where $c$ is a constant as discussed in Pope (2000). The simulation data of Spalart (1988) were used for the inner portion of the inner layer and the experimental data of DeGraaff \& Eaton (2000) were used for the outer portion of the inner layer (because of the low-Reynolds-number effects of the Spalart (1988) data in this outer portion). It is important to note that this is simply an empirical fit of experimental and simulation data, and the specific values of the constants are not of great importance. The important concept here is an inner layer that is universal for all zero-pressure-gradient smooth-wall boundary layers.

The outer portion of the formulation is given by incorporating a viscous and wake deviation to the asymptotic form of the existing log-region formulation

$$
f_{O 3}\left[z^{+}, \delta^{+}\right]=A_{3}-V_{g 3}\left[z^{+}, \frac{z}{\delta}\right]-W_{g 3}\left[\frac{z}{\delta}\right] .
$$

Following Hafez (1991), $A_{3}=1.78$. The viscous correction term has the same isotropic eddy cutoff as the streamwise formulation

$$
V_{g 3}\left[z^{+}, \frac{z}{\delta}\right]=V\left[z^{+}\right]\left(1-\frac{z}{\delta}\right) .
$$

The viscous deviation, $V_{g 3}$, is composed of only an isotropic eddy cutoff. Similar to the streamwise formulation, it is expected that there will also be an anisotropic contribution; however, because of the lack of accurate high-resolution wall-normal turbulence-intensity data covering a large range of Reynolds numbers, the correct form of the anisotropic portion is currently unknown. For consistency, the wake deviation is again based on the Lewkowicz (1982) polynomial expression for the wake deviation of the mean flow. Using this expression and forcing the boundary conditions in the inner and outer regions, ${\overline{u_{3}^{2}}}^{+}=0$ and $\partial\left({\overline{u_{3}^{2}}}^{+}\right) / \partial(z / \delta)=0$ at $z / \delta=1$ 
and $W_{g 3}=0$ as $z / \delta \rightarrow 0$ gives

$$
W_{g 3}\left[\frac{z}{\delta}\right]=A_{3}\left(\frac{z}{\delta}\right)^{2}\left(3-2 \frac{z}{\delta}\right) .
$$

The important distinction to notice about this extended formulation is that there is no scaling function as there is for equation (2.1). This is because the asymptotic limit of $f_{\mathrm{O}_{3}}$ is a constant, and therefore, there is no need to scale the inner formulation to match the outer formulation.

It is important to note that for the above formulations, all of the constants are determined at one Reynolds number. These constants are then held fixed when the formulation is calculated for other Reynolds numbers. That is, these formulations are not simply curve fits at each Reynolds number. Their functional forms are founded on the dimensional analysis and spectral similarity arguments of the attached-eddy hypothesis and one data set is used to determine the value of the constants.

\section{Experimental methods}

\subsection{Test site}

The high-Reynolds-number experimental data used in this work were obtained in the surface layer of the atmospheric boundary layer. The atmospheric boundary layer is comprised of several meteorological layers. The innermost meteorological region, called the surface layer $(\sim 100 \mathrm{~m})$ is the part used to model high-Reynolds-number wall-bounded turbulence. Stull (1988), Kaimal \& Finnigan (1994), and Wyngaard (1992) give excellent reviews of the features of the surface layer as well as the entire atmospheric boundary layer. Here, only the specific properties of the surface layer will be discussed insofar as to give an accurate description of the flow and show that, close enough to the wall, under appropriate conditions, it appears to model the turbulent wall region $\left(100<z^{+}<0.15 \delta^{+}\right)$sufficiently in high-Reynolds-number wall-bounded flows.

\subsubsection{SLTEST}

The hot-wire data were obtained from field studies conducted over three summers at the Surface Layer Turbulence and Environmental Science Test (SLTEST) facility located on the southern end of the western Utah salt flats. The salt flats extend over $240 \mathrm{~km}$ north-south and $48 \mathrm{~km}$ east-west and are extremely flat and barren. The elevation varies less than $1 \mathrm{~m}$ over $13 \mathrm{~km}$ north of the site. A good description of the site, as well as a general discussion of the advantages and challenges of running experiments in the atmospheric surface layer, are found in Klewicki, Foss \& Wallace (1998) and Metzger \& Klewicki (2001). All experiments were conducted on or around the Near Surface Turbulence Measurement Platform (NSTMP) developed by the University of Utah's group. The NSTMP consists of a concrete pit $(\approx 0.6 \mathrm{~m}$ deep) that has a frame that holds $0.61 \mathrm{~m} \times 1.2 \mathrm{~m}$ ultraviolet-resistant polyethylene tiles. The frame and tiles are adjusted so that the tops of the tiles are level with the surface of the salt flats. The calibration equipment is kept in the pit, under the tiles, which provides for a low wind disturbance area. The view over the closed platform looking north is shown in figure 1 . One of the advantages of testing on the salt flats is the regeneration of the surface of the desert owing to the recession of water that covers the flats in the winter. This leads to a relatively smooth wall with aerodynamic roughness heights in the range $\sim 0.1-10 \mathrm{~mm}$. A more detailed discussion of the roughness is given in $\S 4.1$. 


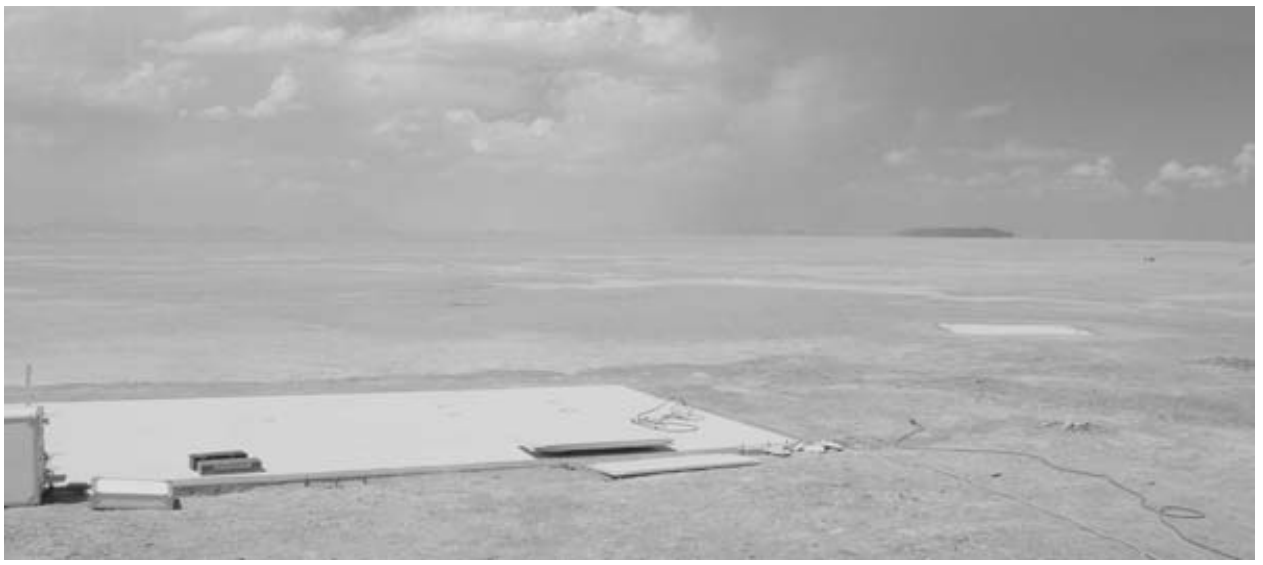

FIGURE 1. View over closed NSTMP.

\subsubsection{Flow scale}

The most significant advantage of using the atmospheric surface layer to model a high-Reynolds-number boundary layer is the extremely large scale of the flow. Since the thickness of the surface layer, which is comparable to the boundary-layer thickness, is large $(\approx 200 \mathrm{~m})$, the smaller scales of the flow can be resolved using conventional measurement techniques. For example, following the approximate analysis of the Kolmogorov scales given by Tennekes \& Lumley (1972), at a Reynolds number of $10^{6}$, an atmospheric surface layer with a thickness of $100 \mathrm{~m}$ and a laboratory boundary layer with a thickness of $0.1 \mathrm{~m}$ would have Kolmogorov lengths of approximately $1 \mathrm{~mm}$ and $0.001 \mathrm{~mm}$, respectively. The typical probe lengths of our hot wires are $l^{+} \approx 15$, which is much smaller than any comparable laboratory high-Reynolds-number wallbounded flow experiment (e.g. Morrison et al. 2004).

\subsubsection{Stability}

One of the challenges of testing in the atmosphere is the effect of buoyancy. To minimize any buoyancy effects, the measurements were taken in the evening when the wall and air temperatures are similar and the surface layer is approaching neutral stability with negligible heat flux to or from the wall.

Quantitatively, the influence of buoyancy in the atmospheric surface layer is typically described by the stability parameter, $\zeta=z / L$, where $L$ is the Obukhov length

$$
L=\frac{-\Theta U_{\tau}^{3}}{\kappa g\left(\overline{u_{3} \theta}\right)_{s}} .
$$

Here, $\Theta$ is the mean potential temperature, $\theta$ is the fluctuating potential temperature, $g$ is the acceleration due to gravity, and the subscript $s$ represents the surface-layer value. The Obukhov length is the buoyancy term in the turbulent kinetic energy equation scaled with $U_{\tau}$ and $z$ and it is an indication of the height at which buoyancy effects become important. Typically, the region in the surface layer at height $z$ is considered stable when $\zeta>0$, unstable when $\zeta<0$, and near-neutral when $|\zeta| \lesssim 0.1$ (e.g. Hogstrom et al. 2002). For this study, $|\zeta|<0.03$ (the individual data set values are given in table 2). 
Monin \& Yaglom (1971) suggest that the non-dimensional gradient of the classic logarithmic mean velocity profile,

$$
\phi_{M}[\zeta]=\frac{\partial U}{\partial z} \frac{\kappa z}{U_{\tau}},
$$

is negligibly affected by buoyancy $\left(\phi_{M}[\zeta]=1\right)$ for $|\zeta| \lesssim 0.03$. Likewise, Foken \& Skeib (1983) suggest and show data supporting $\phi_{M}[\zeta]=1$ for $-0.06 \leqslant \zeta \leqslant 0.13$. However, Hogstrom (1988) shows that even at these small values of the stability parameter, buoyancy does have an effect on the mean velocity gradient and gives the empirical function,

$$
\phi_{M}[\zeta]=1.00+3.43 \zeta+8.4 \zeta^{2}, \quad|\zeta| \leqslant 0.1
$$

Using equation (3.3), the corresponding values of $\phi_{M}$ for the values of the stability parameter for the data used in this study range from 0.93 to 1.11 . Since the focus of this study is turbulence quantities, with due caution, the difference of the mean velocity profile for the nearly neutral boundary layer and the classical log law formulation is taken as negligible. For the turbulence quantities, Pahlow, Parlange \& Porte-Agel (2001) find that the streamwise, spanwise and wall-normal turbulence intensities are negligibly affected by buoyancy for $\zeta \lesssim 0.1$.

The effect of the stability on spectra obtained in the surface layer has been investigated by Kaimal et al. (1972) and Busch (1973). All of the spectra were plotted in the same form as Kaimal et al. (1972) and compared to the expected deviations for buoyancy (not shown). While the wall-normal spectra and cospectra follow the behaviour suggested by Kaimal et al. (1972) at near-neutral conditions, the streamwise spectra do not. However, the Kaimal et al. (1972) model does not take into account the expected change in spectra owing to the wall-normal position or the Reynolds-number, as discussed in Perry et al. (1986), and therefore are not expected to be quantitatively correct across a range of wall-normal positions and Reynolds numbers. Aside from this aspect of the streamwise model, the spectra in this study follow the near-neutral spectral model and are assumed to be negligibly affected by buoyancy. Note, this is consistent with the negligible effects expected on the turbulence intensities (the integrals of the spectra) discussed previously. Therefore, the high-Reynolds-number boundary layers analysed in this study are assumed to be effectively neutral boundary layers with negligible buoyancy effects.

\subsection{Data acquisition}

Single-element hot wires and two-element $x$-probes were used to obtain the instantaneous velocity in the streamwise and wall-normal directions. The wires were arranged in a wall-normal array above the floor of the salt flats ranging from 0.005 to $2.0 \mathrm{~m}$. All wire filaments were $5 \mu \mathrm{m}$ platinum-coated tungsten wires with a $1 \mathrm{~mm}$ sensing length and copper-plated stubs. Typically, ten hot wires were operated with an AA Labs 10-channel anemometer and four were operated with a TSI IFA-100 four-channel anemometer. All wires were run at an overheat ratio of 1.7. All signals, including the calibration temperature and Pitot-static pressure, were conditioned with a Tektronix VX4780 signal conditioner and digitized with a Tektronix VX4244 16-bit resolution digitizer. During data collection on the salt flats, all wires were low-pass filtered at $5 \mathrm{kHz}$ and simultaneously sampled at a rate of $10 \mathrm{kHz}$, typically for $30 \mathrm{~min}$. Note that while this is not long enough to fully resolve all of the larger scales in the flow, it is long enough to begin to see a levelling off of the energy content of the velocity fluctuations at low wavenumbers, as shown in figure 2. Any significantly longer sampling time would begin to include energy from the very low-frequency 


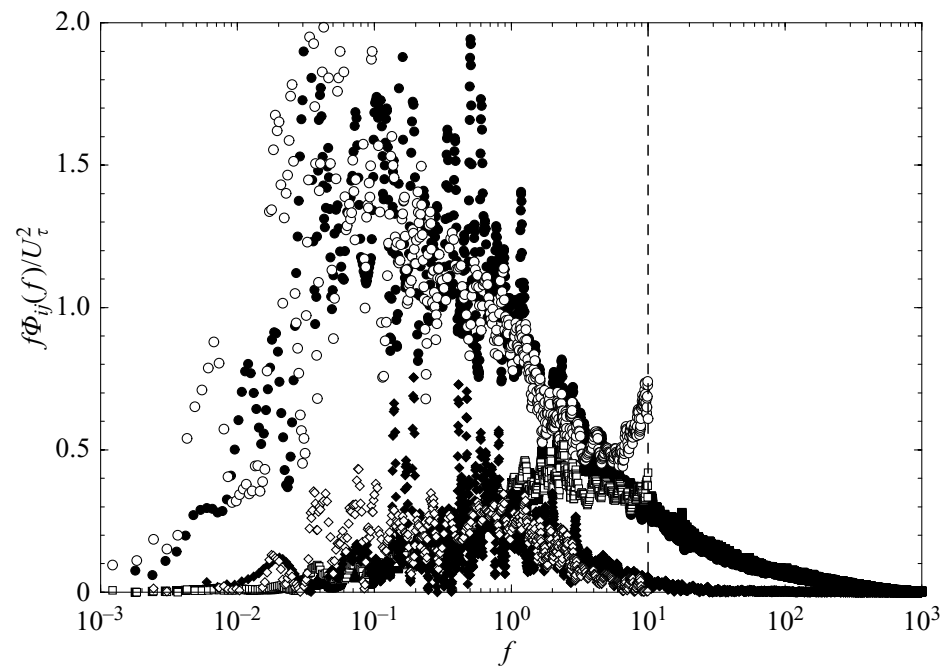

FiguRE 2. Cross-wire probe and sonic anemometer energy spectra comparison. Circles and squares are streamwise $\left(\phi_{11}\right)$ and wall-normal $\left(\phi_{33}\right)$ velocity spectra, respectively. Diamonds are cospectra $\left(-\phi_{13}\right)$. Filled symbols are hot-wire data and open symbols are sonic data. The dashed line is the sonic anemometer sampling limit based on the Nyquist limit $(10 \mathrm{~Hz})$.

diurnal wind changes. Lumley \& Panofsky (1964) show a schematic of the wind speed spectrum near the ground in the atmospheric surface layer, clearly showing the separation of time scales between local (minutes) and diurnal wind fluctuations. It also shows that the transition region between the two, where the fluctuating energy is low, is of the order of an hour. The alternative and ideal method is to acquire many records under identical conditions and ensemble average them. However, this depends on being able to obtain records over many days with identical conditions, or have many arrays at different spanwise positions. Neither of these were feasible for the present study.

All wires were calibrated in a purpose-built calibration facility before and after each experimental run. The calibration facility used ambient air. A static polar lookup-table $\times$-probe calibration technique based on the work of Gresko (1988) was used. The $\times$-probes were typically calibrated at nine angles in the range $\pm 32^{\circ}$ for each of seven velocities ranging from $1-13 \mathrm{~m} \mathrm{~s}^{-1}$. The hot wires were calibrated at the same time as the $\times$-probes at each zero angle location. The probes were calibrated against a Pitot-static tube mounted in the calibration tunnel. The differential pressure was measured with a 10 Torr MKS 698A11TRA pressure transducer that was operated by a MKS 670B signal conditioner. The pressure transducer has an accuracy of \pm 0.05 of the reading and the signal conditioner has an analogue signal output accuracy of $\pm 0.003 \mathrm{~V}$ (which corresponds to $0.4 \mathrm{~Pa}$ ). The temperature of the calibration flow was measured with a Cole-Parmer 4-wire single-element resistance temperature detector (RTD). The RTD was held in an air/gas sheath and was connected to a Cole-Parmer $1 / 8$ DIN RTD Panel Meter that has an accuracy of $\pm 0.2^{\circ} \mathrm{C}$. The temperature during data acquisition was measured by the University of Utah group with 4-5 quartz thermometers placed in a wall-normal array $0-1 \mathrm{~m}$ above the salt flats.

During any single calibration and experimental run, the maximum temperature change was typically less than $1^{\circ} \mathrm{C}$ with the standard deviation of the temperature being lower $\left(\approx 0.3^{\circ} \mathrm{C}\right)$. These small variations (during a single calibration and a single 
acquisition period) are assumed to affect both the calibration and acquisition results negligibly. However, because of the larger temperature difference between each data acquisition period and starting and ending calibrations $\left(\approx 5-10^{\circ} \mathrm{C}\right)$, all calibrations were interpolated as a function of temperature (using the starting and ending calibrations) to obtain a calibration surface valid for the temperature at which the data were acquired. Specific details on the interpolation of the calibration surfaces as a function of temperature can be found in Kunkel (2003). An independent laboratory investigation was conducted to test the temperature interpolation method over the range of temperatures experienced in Utah. The interpolation method was tested by comparing the calibration velocities and turbulence statistics from interpolated calibrations to actual calibrations conducted at the data acquisition temperatures. Over the range of temperatures and velocities measured in the atmosphere during near-neutral conditions, the relative error with respect to the mean velocity due to the interpolation is estimated to be less than $3 \%$. The standard deviation, skewness and flatness of a turbulent signal are accurate to $2 \%$ of the respective measured quantity. Overall, the simple interpolation method is found to be acceptably accurate and is preferred over either classical extrapolation methods or more complex methods where the use of a cold wire and extended temperature calibration is required.

A simpler alternative to using hot wires is to use sonic anemometers, which are a popular experimental tool in atmospheric studies. However, for this study, in which it is desired to resolve all the energy-containing scales in the flow and measure relatively close to the wall, the sonic anemometers with a measuring path of approximately $10 \mathrm{~cm}$ and a sampling rate of approximately $20 \mathrm{~Hz}$ are too large and sample too slowly. The difference in the streamwise and wall-normal fluctuating energy content between a sonic anemometer and a hot wire, as well as the difference in the cospectrum, is seen in figure 2. Here, for the streamwise and wall-normal spectra, the sonic anemometer does not resolve all the energy-containing scales of the flow and also aliases the higherfrequency energy. Note, however, that while the streamwise and wall-normal spectra are not completely resolved, the cospectrum is almost completely resolved. This is consistent with the theoretical arguments which suggest that the cospectrum decay more quickly (with a $-7 / 3$ slope) than the streamwise or wall-normal spectra $(-5 / 3$ slope) at high frequencies. This suggests that it is possible to measure Reynolds shear stress, in this flow, using sonic anemometers. This is important because the Obukhov length, which depends on the Reynolds shear stress through the friction velocity, and thus the stability parameter, were calculated using sonic anemometers.

\subsection{Data qualification}

Unlike laboratory experiments where mean-flow parameters (e.g. free-stream velocity and streamwise pressure gradient) are typically set to produce a desired boundary layer, experiments in the field afford no such luxury. Because of the uncontrollable elements in the atmospheric boundary layer, each individual data set must be scrutinized and qualified in terms of the mean characteristics of the flow. Therefore, a qualification procedure was used to eliminate the data which do not appear to have been acquired in a boundary layer representative of a canonical turbulent boundary layer. First, only near-neutral $(|\zeta| \lesssim 0.1)$ data are used. Secondly, spanwise flow direction fluctuations from sonic anemometers are analysed for each corresponding hot-wire data set. Any data acquired during time periods with large wind shifts greater than $\pm 20^{\circ}$ are not used. Thirdly, filtered velocity signatures with a filter size of approximately the boundary-layer thickness, autocorrelations and probability density functions are calculated and compared to the description of stationary data 


$\begin{array}{lccccc}\text { Data set } & U_{\tau}^{2}(\%) & U^{+}(\%) & \overline{u_{1}^{2}}(\%) & \overline{u_{3}^{2}}(\%) & -\overline{u_{1} u_{3}}+(\%) \\ \text { 14 August 21:07 2000 } & 5 & 6 & 11-16 & 6-9 & 7-16 \\ \text { 14 August 21:46 2000 } & 5 & 7 & 7-11 & 6-9 & 7-16 \\ \text { 9 July 19:23 2002 } & 5 & 6 & 11-21 & 7-11 & 7-21 \\ \text { 9 July } 20: 072002 & 5 & 6 & 11-16 & 7-11 & 7-26\end{array}$

TABLE 1. Estimated total error for atmospheric data using the root-sum-square method suggested by Coleman \& Steele (1989). $U_{\tau}^{2}$ is calculated from the $\overline{-u_{1} u_{3}}$ value closest to wall.

given by Bendat $\&$ Piersol (2000). Any data which appear to be non-stationary are not used.

The total time over which the hot-wire data were acquired, close to neutral conditions, over the three field trials was $48 \mathrm{~h}$. After the qualification procedure, a total of $2 \mathrm{~h}$ of the data are considered high enough quality to be used in this study. From the qualification procedure, it was observed that the 2002 data sets contain some slight non-stationary effects, and thus this data set is considered to be less descriptive of canonical wall-bounded turbulence. However, to show that the atmospheric results from 2000 are not an anomaly, the 2002 data are also shown.

\subsection{Data uncertainty}

Wall-normal positions $z \leqslant 150 \mathrm{~mm}$ were measured with a machinist's rule and the expected maximum uncertainty for these values is $\pm 0.05 \mathrm{~mm}$. For all wall-normal measurements $z>150 \mathrm{~mm}$, a tape measure was used and the expected maximum uncertainty in the wall-normal position is $\pm 2 \mathrm{~mm}$. When plotted on a log scale, this leads to negligible differences for all of the profiles.

The main sources of error for the velocity statistics come from the propagation of systematic instrumentation errors and the incomplete convergence due to the limited sampling time. However, unlike in the laboratory, the errors from incomplete convergence are much larger than the propagation of systematic errors. Therefore, since standard propagation of errors is not applicable to the look-up-matrix calibration for the $\times$-probes, the error estimates of Yavuzkurt (1984), are used for the systematic errors. Following Yavuzkurt (1984), we take the systematic errors for the single component hot-wire data for $\overline{u_{1}^{2}}$ and $U$ to be $1 \%$. For the $\times$-probes (which Bruun, 1995, suggests yields the same results as the Yavuzkurt, 1984, 3-wire probe) we use errors of $2 \%$ for $\overline{u_{1}^{2}}$ and $U$, and $4 \%$ for $\overline{u_{3}^{2}}$.

The most significant source of error in most of the statistics is the error that results from incomplete convergence due to the limited sampling time and changing large-scale weather patterns which makes ensemble averaging impossible. The size of the last large-scale oscillation in the running averages of $U, \overline{u_{1}^{2}}, \overline{u_{3}^{2}}$, and $\overline{-u_{1} u_{3}}$ were used to estimate the error due to the lack of convergence. Using this method, we find convergence errors of $\sim 5-20 \%$ with $\overline{u_{1}^{2}}$ having the largest uncertainty. These results are consistent with those obtained from the theoretical ensemble-average difference suggested by Wyngaard (1992) for a 30 min average.

Combining the systematic instrumentation errors, the calibration interpolation errors (discussed in \$3.2), and the convergence errors using the root-sum-square method as described by Coleman \& Steele (1989), the maximum expected uncertainties for the data used in this paper are given in table 1. These results are consistent with the spread in the experimental results given in the next section. Specifically, notice 


\begin{tabular}{lccccc} 
& \multicolumn{2}{c}{14 August 2000} & & \multicolumn{2}{c}{9 July 2002} \\
\cline { 2 - 3 } \cline { 5 - 6 } Symbol & $21: 07$ MDT & $21: 46 \mathrm{MDT}$ & & $19: 23 \mathrm{MDT}$ & $20: 07 \mathrm{MDT}$ \\
$\delta^{+}$ & $\bullet$ & $\mathbf{0}$ & & $\bullet$ & $\boldsymbol{\Delta}$ \\
$U_{\tau}\left(\mathrm{m} \mathrm{s}^{-1}\right)$ & $3.1 \times 10^{6}$ & $3.8 \times 10^{6}$ & & $2.3 \times 10^{6}$ & $2.3 \times 10^{6}$ \\
$\delta(\mathrm{m})$ & 0.287 & 0.355 & & 0.222 & 0.215 \\
$k_{s}(\mathrm{~mm})$ & 200 & 200 & & 200 & 200 \\
$\Delta U^{+}$ & 15.9 & 15.9 & & 2.9 & 2.9 \\
$\nu$ & 9.9 & 10.5 & & 4.6 & 4.6 \\
$U_{\tau}(\mathrm{mm})$ & 0.065 & 0.052 & & 0.086 & 0.088 \\
$L(\mathrm{~m})$ & 60 & 90 & & -115 & 85 \\
$\zeta(z=2 \mathrm{~m})$ & 0.03 & 0.02 & & -0.02 & 0.02
\end{tabular}

TABLE 2. Mean-flow parameters.

that there are higher uncertainties for the 9 July 2002 data, as expected from the qualification procedures. These results are also consistent with the errors listed by Metzger \& Klewicki (2001) that were obtained from data acquired in a similar experimental atmospheric-boundary-layer study.

\subsection{Mean-flow parameters}

Table 2 gives the calculated and assumed mean-flow parameters of the data sets used in this study. The boundary-layer thickness is taken to be $200 \mathrm{~m}$ for all data sets. This assumption is based on similar days' miniSODAR and rawinsondes mean velocity profiles during near-neutral stability at the SLTEST site as reported by Klewicki et al. (1998) and Hommema \& Adrian (2003). Klewicki et al. (1998) show two typical atmospheric-boundary-layer mean-velocity profiles (their figure 1) where it is easy to see the significant change in the mean velocity (both magnitude and direction) that occurs around $200 \mathrm{~m}$. Any implications of this assumption will be discussed where necessary. The friction velocity was calculated from the Reynolds shear stress $\left(U_{\tau}=\left(-\overline{u_{1} u_{3}}\right)^{1 / 2}\right)$ at the closest position above the wall within the mean velocity log region. During near-neutral stability, Metzger (2002) found this method of calculating the friction velocity agrees well with several other methods, including direct measurement using a drag plate. This also agrees with Weber (1999) who suggests that over simple terrain, in near-neutral conditions, when aligned with the mean flow, $\left(-\overline{u_{1} u_{3}}\right)^{1 / 2}$ is an accurate estimator of $U_{\tau}$.

\section{Results and discussion}

\subsection{Mean-flow similarity}

The mean velocity measurements in the streamwise direction, scaled with inner-flow variables $\left(U^{+}=U / U_{\tau}, z^{+}=z U_{\tau} / \nu\right)$ are shown in figure 3 . Here, the shift in the logarithmic mean velocity profile owing to roughness is easily seen. It is generally accepted (e.g. Schlichting 1968) that the mean velocity profile of the zero-pressuregradient neutral turbulent boundary layer follows

$$
U^{+}=\frac{1}{\kappa} \ln \left[\frac{z}{k_{s}}\right]+B,
$$




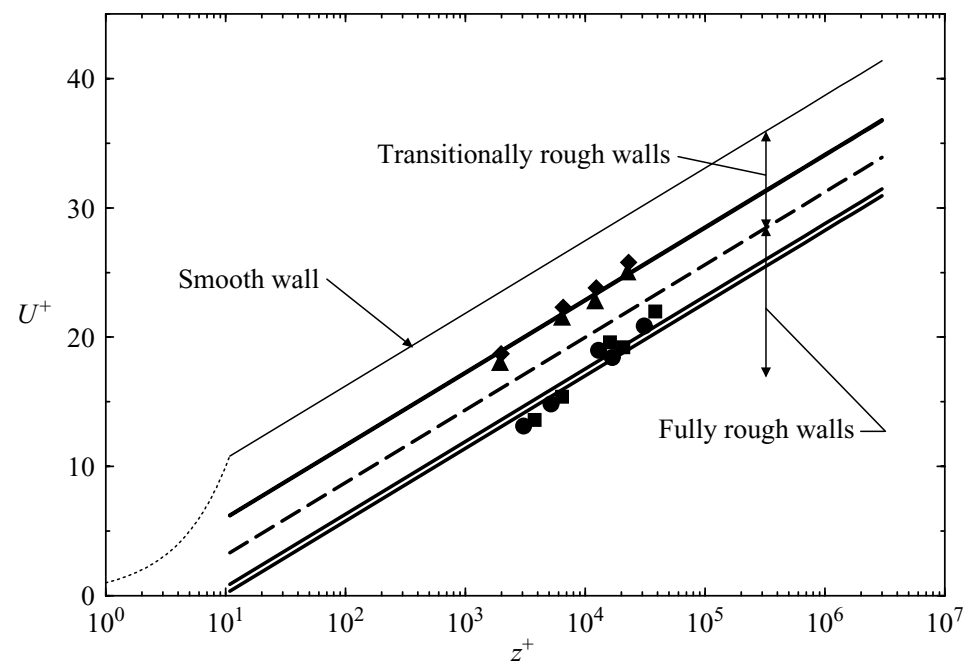

FiguRE 3. Mean-flow similarity. Symbols as in table 2.

where $\kappa$ is the Kármán constant (taken to be 0.41 ), $k_{s}$ is the equivalent sand grain roughness height, and $B$ is the intercept, which is a function of the roughness height. Following Ligrani \& Moffat (1986) for hydrodynamically smooth walls $\left(k_{s}^{+}<2.25\right)$,

$$
B=\frac{1}{\kappa} \ln \left[k_{s}^{+}\right]+A,
$$

where $A$ is the classic smooth-wall log law intercept (taken to be 5.0). For fully rough walls $\left(k_{s}^{+}>90\right)$,

$$
B=8.5 \text {. }
$$

In the transition region $\left(2.25 \leqslant k_{s}^{+} \leqslant 90\right)$,

$$
B=\frac{1}{\kappa} \ln \left[k_{s}^{+}\right]+A+\left(8.5-A-\frac{1}{\kappa} \ln \left[k_{s}^{+}\right]\right) \sin \left[\frac{1}{2} \pi h\right]
$$

where

$$
h=\frac{\ln \left[k_{s}^{+} / 2.25\right]}{\ln [90 / 2.25]} .
$$

The equivalent sand grain roughness height for the fully rough wall (2000) and transitionally rough wall (2002) data are found by least-squares-error curve-fits to equation (4.2) with $B$ defined by equation (4.3) and equation (4.4), respectively. The roughness heights are shown in table 2. Qualitatively, the equivalent sand grain roughness height is similar to the actual roughness height observed on the salt flats. Using this representation, the Hama (1954) roughness function (the rough-wall deviation from the smooth-wall log law) is then

$$
\Delta U^{+}=\frac{1}{\kappa} \ln \left[k_{s}^{+}\right]+A-B .
$$

\subsection{Turbulence intensities}

The streamwise turbulence intensities $\left({\overline{u_{1}^{2}}}^{+}=\overline{u_{1}^{2}} / U_{\tau}^{2}\right)$ from the atmospheric surface layer with inner- and outer-flow scaling are shown in figure 4. For comparison, the simulation data of Spalart (1988), the high-resolution experimental data of 


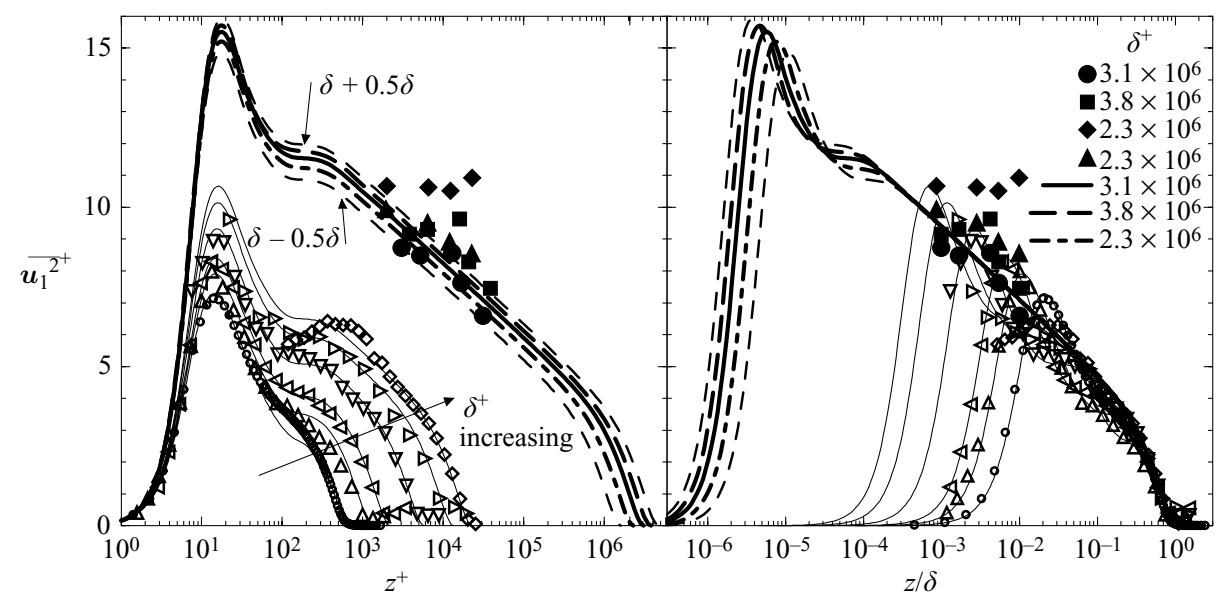

Figure 4. Streamwise turbulence intensities. Solid symbols are atmospheric data. Open symbols are laboratory data; $\delta^{+}=689(\bigcirc)-S p a l a r t(1988) ; 1335(\triangle), 2217(\triangleleft), 5813(\nabla)$, $13490(\triangleright)$-DeGraaff \& Eaton (2000); $23013(\diamond)-$ Fernholz et al. (1995). Solid, dashed and dot-dashed lines are smooth-wall similarity formulations (equation (2.1)) that are also valid for rough walls in the outer region. Lighter dashed lines are similarity formulation for $\delta^{+}=3.1 \times 10^{6}$ if boundary-layer thickness is $50 \%$ larger or smaller.

DeGraaff \& Eaton (2000), and the high-Reynolds-number laboratory data of Fernholz et al. (1995) are shown. The corresponding streamwise turbulence-intensity similarity formulations (equation (2.1)) are also shown for all data. Recall the inner portion of the formulation is valid only for smooth walls, but the outer part is the Marusic et al. (1997) similarity formulation, which is applicable to both smooth and rough walls. Here, the entire formulation is given to stress that the expected peak in the smooth-wall streamwise turbulence intensity does increase with increasing Reynolds numbers. This outer-flow influence, which increases with the Reynolds number, is felt all the way down to the wall. This suggests the inner portion of the boundary layer is not completely autonomous (i.e. Waleffe 1997; Jiménez \& Pinelli 1999). As in Marusic et al. (1997), the formulation is seen to describe the laboratory range Reynolds numbers well, as well as the extremely large-Reynolds-number atmospheric data. The similarity formulation for a Reynolds number of $3.1 \times 10^{6}$ is also shown if the boundary-layer thickness was $50 \%$ thicker $\left(4.7 \times 10^{6}\right)$ or thinner $\left(1.6 \times 10^{6}\right)$. Again, it is important to note that while there is a relatively high uncertainty in the atmospheric data (as compared to laboratory and computational studies), the data show that the level of streamwise turbulence intensity in the log region of the flow is increasing with the Reynolds number and is generally described by the similarity formulation. Marusic et al. (2004) also find the modified streamwise formulation to describe high-Reynolds-number pipe flow well, agreeing with Morrison et al. (2004) that the outer-flow influence in pipe flow also increases with Reynolds number.

The wall-normal turbulence intensities are shown in figure 5 along with the corresponding similarity formulations given in $\S 2$. Again, the inner portion of the formulation is valid only for smooth walls, but the outer part is hypothesized to be valid for both smooth and rough walls. Recall that in the log region, the outer portion tends to the asymptotic high-Reynolds-number form of

$$
{\overline{u_{3}^{2}}}^{+}=A_{3} .
$$




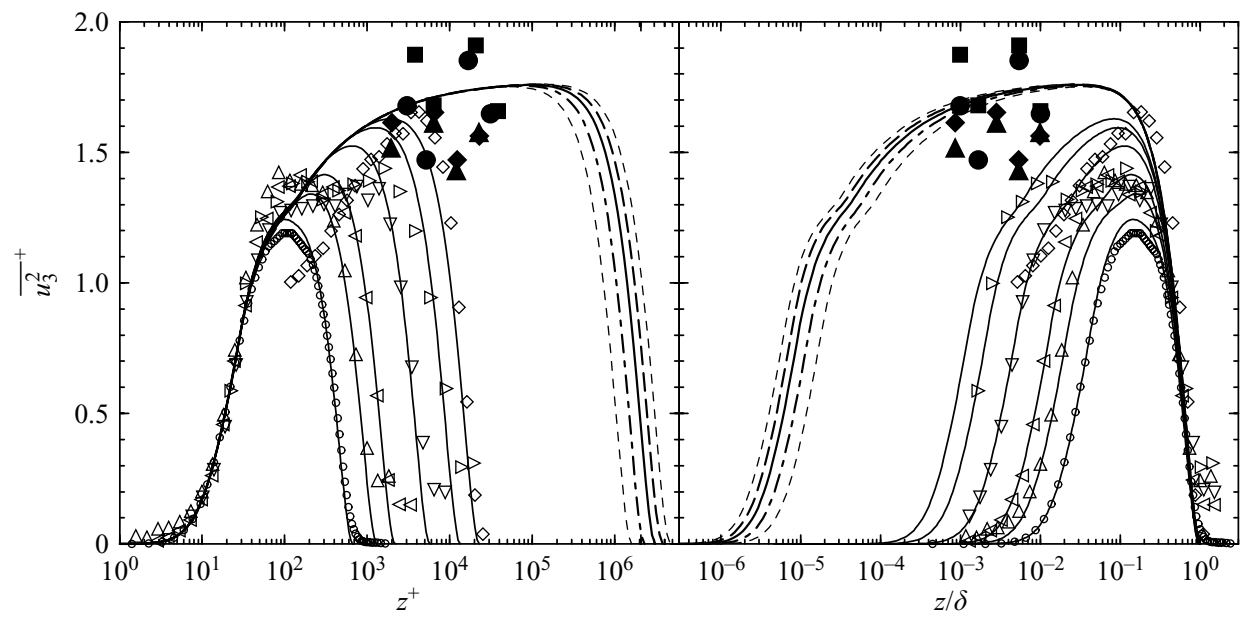

FIGURE 5. Wall-normal turbulence intensities. Symbols as in figure 4.

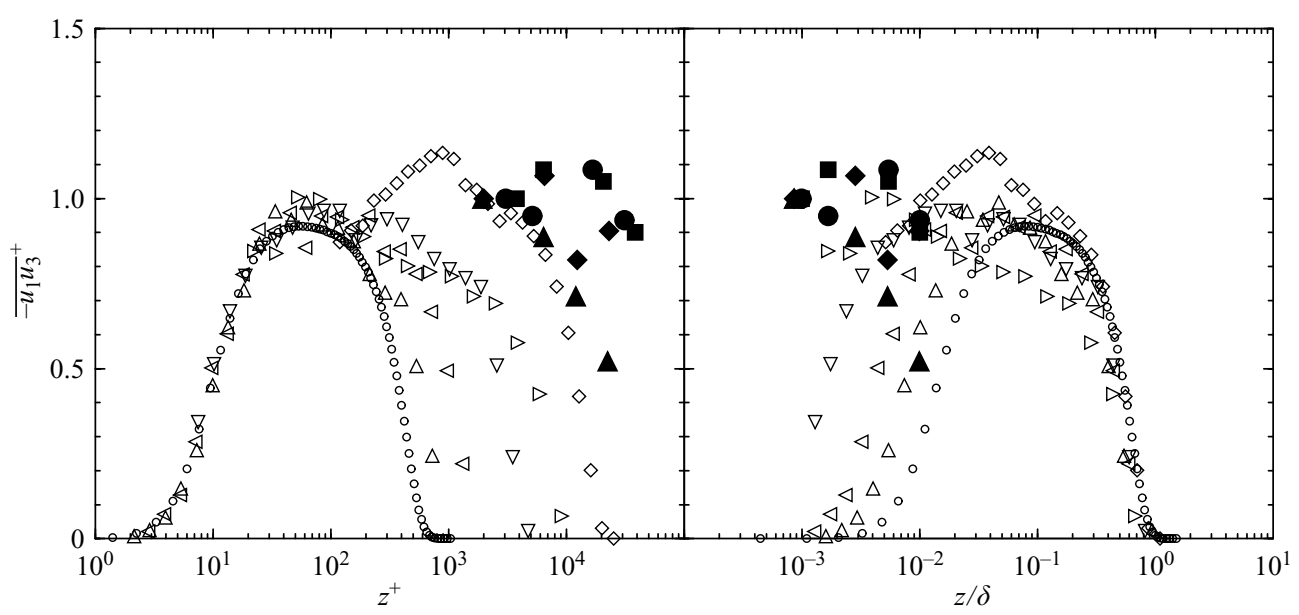

FigurE 6. Reynolds shear stresses. Symbols as in figure 4.

The wall-normal similarity formulation, which is again based on the attached-eddy model, also appears to describe the high-Reynolds-number data well. That is, there is no definite increase with Reynolds number and all of the data are arbitrarily scattered around the formulation. This is regarded as significant since the constants determined from laboratory data $\left(\delta^{+} \sim 10^{3}\right)$ do indeed seem to apply at high Reynolds numbers $\left(\delta^{+} \sim 10^{6}\right)$.

The Reynolds-shear-stress profiles are shown in figure 6. With the exception of the slightly non-stationary 9 July 2002, 19:23 data set (as described in $\S 3.3$ ), the shear stresses behave as expected for high-Reynolds-number flow suggesting an extended range of nearly constant shear stress in the log region. These data are also consistent with Reynolds stress analysis given by Priyadarshana \& Klewicki (2004).

In general, within the accuracy of the data, the similarity formulations seem to describe the data well from laboratory to atmospheric range Reynolds numbers. This is in agreement with Townsend's (1956) Reynolds-number similarity hypothesis. That is, at sufficiently high Reynolds-numbers, the energy-containing motions (turbulence 
intensities) are independent of viscosity (and roughness) when scaled with outer-flow variables, insofar as the viscosity (and roughness) may affect the boundary conditions $\left(U_{\tau}\right)$.

\subsubsection{Roughness effects}

Krogstad \& Antonia (1999) have suggested that Townsend's Reynolds-number similarity hypothesis is not valid above rough-wall boundary layers. This conclusion was based on experiments conducted above smooth- and rough-wall boundary layers with similar mean-flow parameters. Krogstad \& Antonia found that while their streamwise turbulence intensities were similar, the wall-normal turbulence intensities for the rough wall showed a distinct increase $(\sim 40-60 \%)$ over the smooth-wall results across most of the layer. They suggest this difference is due to the roughness and hence violates Townsend's Reynolds-number similarity hypothesis. Bhaganagar et al. (2004) conducted a direct numerical simulation of a turbulent channel flow with roughness on one wall and similarly conclude that outer-layer similarity is not valid above rough walls. However, several other studies, such as Perry \& Li (1990) and Flack et al. (2005) have found that both smooth- and rough-wall streamwise and wall-normal turbulence intensities do agree, and that the major local effect of the roughness is simply to increase the shear stress at the wall, in agreement with Townsend's hypothesis. Similarly, the attached-eddy model supposes that the main effect of roughness (aside from increasing the wall shear stress) is to set the smallest attached-eddy scale (Perry \& Li 1990).

Our rough-wall atmospheric results are consistent with the smooth-wall similarity formulations (i.e. they show no definite increase or trend with roughness and all lie arbitrarily within $20 \%$ of the formulations). The general agreement with the formulations suggests that the turbulence intensities are similar with outer-flow scaling supporting Townsend's Reynolds-number similarity hypothesis and the attached-eddy model. Similarly, the study of the motions contributing to the Reynolds shear stress by Priyadarshana \& Klewicki (2004) finds that roughness effects the streamwise and wallnormal spectra very little necessitating little effect on the turbulence intensities. Kunkel (2003) suggests the discrepancy arises because the roughness height in Krogstad \& Antonia (1999) was too high with respect to the large outer scales of the flow (the boundary-layer thickness) causing a bluff-body effect rather than a surface roughness effect. For instance, while the roughness heights with inner-flow scaling are similar in both our atmosphere experiment and their laboratory experiment $\left(k_{s}^{+} \approx 240\right.$ and $k_{s}^{+} \approx 380$, respectively), with outer-flow scaling, the heights are very different $\left(k_{s} / \delta \approx 0.008 \%\right.$ and $k_{s} / \delta \approx 7 \%$, respectively). This is in agreement with Jiménez (2004) who suggests that the effects of wall roughness are really dependent on two parameters, $k^{+}$and $k / \delta$ (here $k$ is the physical height of the roughness elements). Jiménez (2004) suggests $k / \delta \lesssim 1-3 \%$ and $\delta^{+}>4000$ before outer-flow similarity can be expected. That is, it is important that both the roughness height and the viscous scale $\left(\nu / U_{\tau}\right)$ be small compared with the outer-flow scale $(\delta)$. This would not be the case in the low-Reynolds-number simulation data of Bhaganagar et al. (2004), and therefore may be the reason for the disparity of their results and the classic scaling. Further experimental work is required in facilities that can reach a continuous range of high enough Reynolds numbers to investigate completely the values of $k / \delta$ and $\delta^{+}$ for which outer-flow similarity applies.

\subsection{Spectra}

The previous similarity formulations are all based on the one-dimensional spectral similarity arguments derived from the attached-eddy model. Good illustrative 


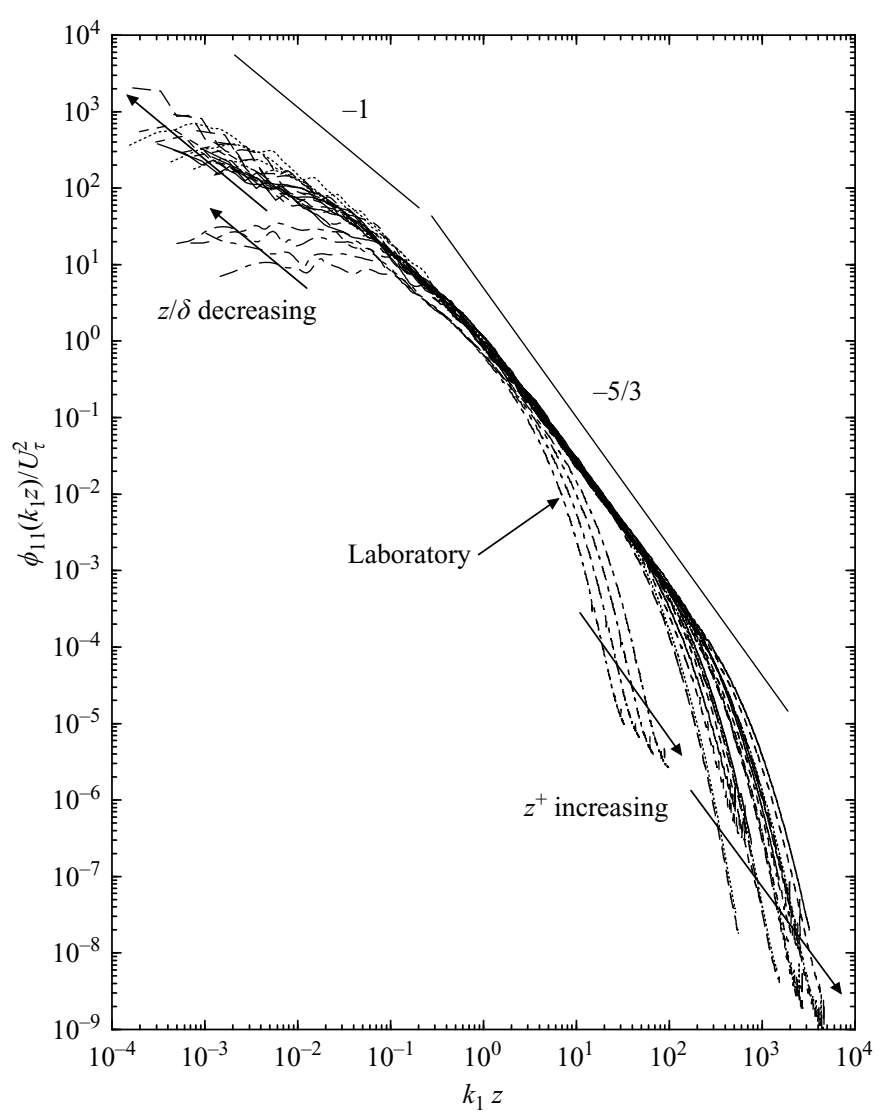

FiguRE 7. Streamwise spectra with inner-flow scaling. Dashed lines are 14 August 21:07, solid lines are 14 August 21:46, dotted lines are 9 July 19:23, and long-dashed lines are 9 July 20:07 data sets. Dot-dashed lines are laboratory hot-wire data for $\delta^{+} \sim 4 \times 10^{3}$.

examples of the expected similarity regions for streamwise and wall-normal spectra are given in Perry et al. (1986) and Perry \& Li (1990). Here, the atmospheric spectra are shown with inner-flow and outer-flow scaling for comparison with the spectral similarity hypotheses.

The spectra were calculated using standard fast-Fourier-transform methods, and varying length ensemble averages similar to the method described in Bendat \& Piersol (2000). Because of poor convergence at low and moderate wavenumbers, additional frequency averaging was also performed following Bendat \& Piersol (2000). Taylor's hypothesis of frozen turbulence was used with the local mean velocity to convert from frequency to wavenumber. Full details of the spectra calculation procedure are given in Kunkel (2003).

The streamwise and wall-normal atmospheric spectra and Reynolds-shear-stress spectra (cospectra) with inner-flow scaling are shown in figures 7, 8 and 9 , respectively. For comparison, laboratory streamwise spectra at $\delta^{+} \sim 4 \times 10^{3}$ and wall-normal and cospectra at $\delta^{+} \sim 1 \times 10^{3}$ are also included. The atmospheric spectra show a significant length of inertial subrange with a $-5 / 3$ behaviour in the streamwise and wall-normal spectra. In agreement with Lumley (1967) and others (Wyngaard \& Cote 1972; Pope 2000 , for instance), the cospectra in the inertial subrange decay more rapidly than the streamwise and wall-normal spectra, following a $-7 / 3$ behaviour. 


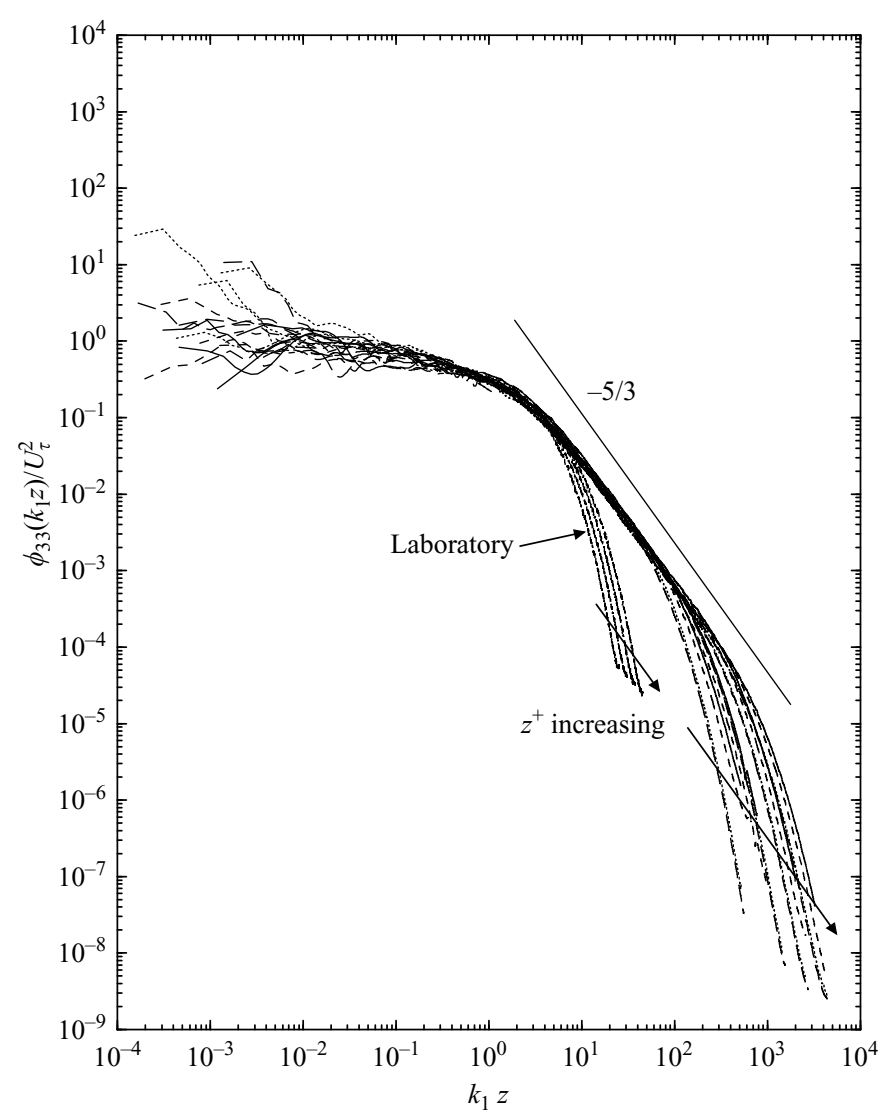

FIGURE 8. Wall-normal spectra with inner-flow scaling. Dashed lines are 14 August 21:07, solid lines are 14 August 21:46, dotted lines are 9 July 19:23, and long-dashed lines are 9 July 20:07 data sets. Dot-dashed lines are laboratory data from Marusic, Kunkel \& Porte-Agel (2001) $\left(\delta^{+} \sim 1 \times 10^{3}\right)$.

According to the attached-eddy model of wall turbulence, with inner-flow scaling, the streamwise, wall-normal and Reynolds-shear-stress spectra should collapse at moderate wavenumbers $\left(k_{1} z \sim 1\right)$. The wall-normal spectra and cospectra should also collapse at low wavenumbers and while some spread is seen, there is actually very little energy at these low wavenumbers (see figures 12 and 13). Overall, the spectra seem to agree fairly well with the scaling hypothesized from the attached-eddy model, at least when viewed in this $\log -\log$ representation. A more critical evaluation is obtained by considering the spectra in a pre-multiplied form with log-linear axes. In this case, equal areas underneath the curve represent equal contributions to the spectral energy, and any -1 region will appear as a horizontal line. Also, with the log-linear plot, any expected collapse on the ordinate can be examined more closely.

\subsubsection{Streamwise spectra}

The pre-multiplied streamwise spectra with outer- and inner-flow scaling are shown in figures $10(a)$ and $10(b)$, respectively. For clarity only the 14 August 21:46, 9 July 20:07, and laboratory data sets are shown. According to the attached-eddy model (i.e. Perry \& Li 1990) the streamwise spectra with outer-flow scaling (figure 10a) should collapse at low non-dimensional wavenumbers and in the hypothesized -1 overlap 


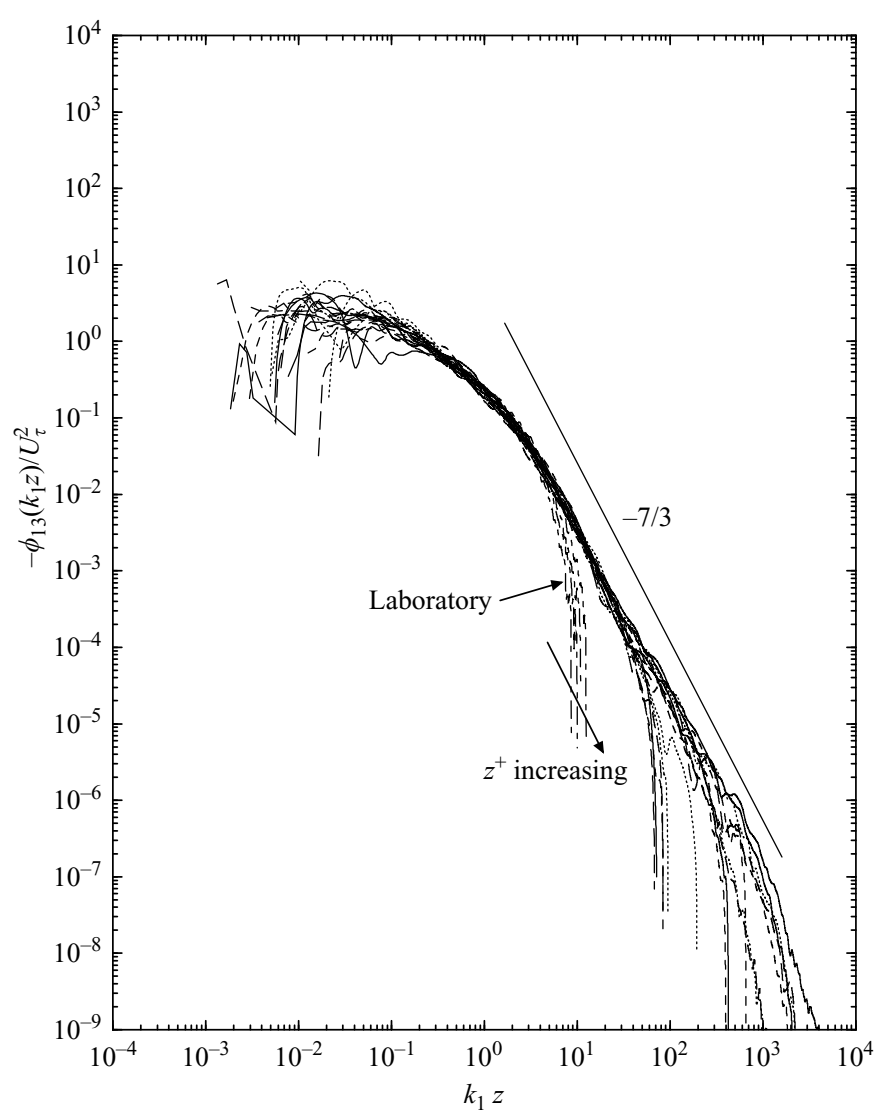

FiguRE 9. Cospectra with inner-flow scaling. Dashed lines are 14 August 21:07, solid lines are 14 August 21:46, dotted lines are 9 July 19:23, and long-dashed lines are 9 July 20:07 data sets. Dot-dashed lines are laboratory data from Marusic et al. $(2001)\left(\delta^{+} \sim 1 \times 10^{3}\right)$.

region. At the high wavenumber end of the -1 region, the spectra should peel off with decreasing $z / \delta$. With inner-flow scaling (figure $10 b$ ), the spectra are expected to collapse at moderate non-dimensional wavenumbers and in the -1 and $-5 / 3$ overlap regions. At wavenumbers lower than the -1 region the spectra should peel off with decreasing $z / \delta$, and at wavenumbers higher than the $-5 / 3$ region the spectra should peel off with increasing $z^{+}$.

The spectra in figure 10 do show some of the expected trends from the attachededdy model, but in general there are clear discrepancies, particularly between the low-Reynolds-number laboratory data and the atmospheric data at low and moderate wavenumbers. The agreements in the trends include: some assemblage with outerflow scaling at low wavenumbers and in the supposed -1 region (particularly in contrast to figure $12(a)$ where no similarity is expected). Also the spectra peel off with decreasing $z / \delta$ with outer-flow scaling at the high-wavenumber end of the supposed -1 region, and at moderate wavenumbers $\left(k_{1} z \sim 1\right)$ there is a marked improvement in similarity with inner-flow scaling (in comparison with outer-flow scaling). At higher wavenumbers, the spectra are better converged and here inner-flow scaling similarity is evident with the spectra peeling off at the end of the expected $-5 / 3$ region with increasing $z^{+}$(similar to figure $\left.12 b\right)$. 

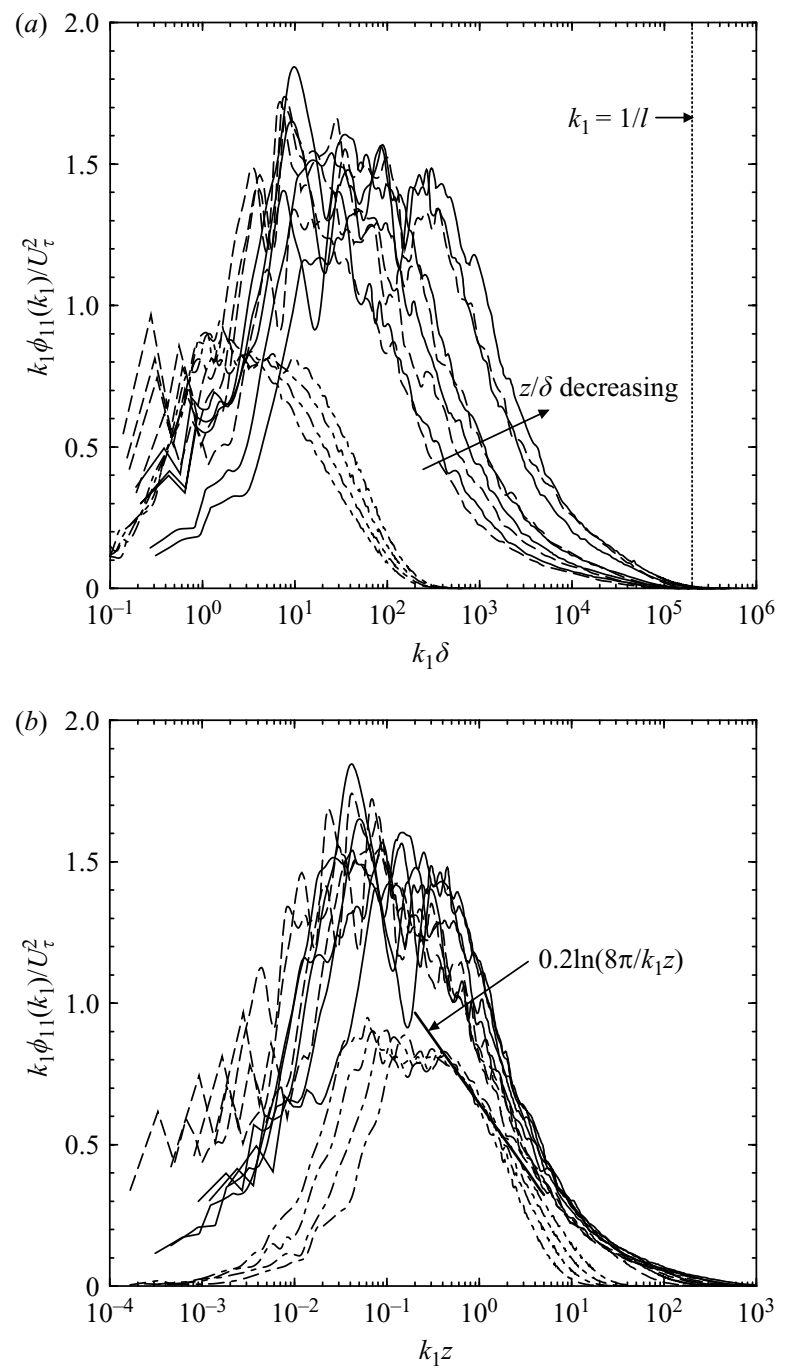

FIGURE 10. Streamwise pre-multiplied spectra. Data as in figure 7. Also shown is the reciprocal of the hot-wire length $(l)$ for the atmospheric data. (a) Outer-flow scaling. (b) Inner-flow scaling.

Quantitatively, however, the expected collapses are poor, and taking the data at face value would indicate that the attached-eddy model is inadequate to describe the streamwise spectra behaviour. One clear difference between the laboratory and atmospheric data are the different levels at which the pre-multiplied spectra approximately plateau (indicating a possible $k_{1}^{-1}$ region). (Note, a -1 region is also consistent with the expected spectral distribution in shear flows given by Tchen (1953), the description of wall turbulence by Jiménez (1999), and the spectral description and verification of the ESL given by Drobinski et al. (2004) and Hunt \& Carlotti (2001)). A possible explanation for the difference in the level of grouping could be that the spectra do not exhibit complete similarity, but rather incomplete similarity as suggested by Morrison et al. (2004) (see also del Alamo et al. 2004). In Morrison et al. (2004), high, $\left(\delta^{+} \sim 10^{5}\right)$ and moderate $\left(\delta^{+} \sim 10^{3}\right)$ Reynolds-number spectra from the Princeton Superpipe are compared and are found not to exhibit complete similarity. 
That is, the spectra do not collapse in the -1 region. Instead, they show distinct peaks at different levels depending on wall-normal position and Reynolds number. However, this study was conducted in a high-Reynolds-number laboratory flow where the turbulent scales are extremely small. Therefore, because of the relatively small boundary layer, the data were obtained over a small range of $z / \delta$, where the smallest $z / \delta=0.030$. As discussed in Marusic \& Perry (1995), it is not clear whether these values of $z / \delta$ are small enough to display the asymptotic -1 power law. From attached-eddy model calculations, Marusic \& Perry (1995) found (their figure 15 and corresponding discussion) that in order to have a reasonable -1 power-law region $z / \delta \lesssim 0.01$. Nickels et al. (2004) performed careful experiments in the new high-Reynolds-number facility at the University of Melbourne and reported evidence of the -1 law at $\delta^{+}=1.6 \times 10^{4}$, albeit for only a short length. Nickels et al. (2004) estimate that for one decade of -1 law to exist $\delta^{+}>5.0 \times 10^{4}$ for $z^{+}=100$. This corresponds to $z / \delta<0.002$.

Strictly, it is difficult to draw any firm conclusions from the the atmospheric data in figure 10 at low and moderate wavenumbers. This is because the largest proportion of energy for the streamwise turbulence intensities are carried at the low and moderate wavenumbers and the data are most probably not converged. This is indicated by the noticeable scatter that is observed (masked further by the frequency averaging). The problem stems from not being able to sample long enough (or collect enough ensembles) in order to obtain converged results.

However, the data shown in figure 10 raise some additional issues. For instance, while the data may not be converged for low and moderate wavenumbers, it can be reasonably assumed that the streamwise spectra are converged at wavenumbers higher than $k_{1} z \sim 1$. According to the spectral model forwarded by Perry \& Li (1990), at this non-dimensional wavenumber, all spectra in the log region are expected to collapse with inner-flow scaling. On a typical log-log plot this would seem to be true. However, careful inspection of figure $10(b)$ shows that this is not strictly the case, and, in fact, a systematic deviation is observed as a function of roughness. To emphasize this trend, a simple integral parameter is defined,

$$
\psi=\int_{1}^{2} \frac{\phi_{11}\left(k_{1} z\right)}{U_{\tau}^{2}} \mathrm{~d}\left(k_{1} z\right) .
$$

Figure 11 shows the integral parameter from equation (4.8) plotted versus the Hama (1954) roughness function $\Delta U^{+}$(equation (4.6)). Here, the ordinate is $\psi^{\prime}$, which is $\psi$ normalized by the corresponding value using the proposed curve-fit for the inner-scaled collapsed region given by del Alamo et al. (2004) (for $2 \pi / 10<k_{1} z<2 \pi$ ), namely

$$
\frac{k_{1} \phi_{11}\left(k_{1}\right)}{U_{\tau}^{2}}=0.2 \ln \left(\frac{8 \pi}{k_{1} z}\right) .
$$

All the atmospheric data are in figure 11, as well as the results from sonic-anemometer spectra taken in 2004 when the salt flats' roughness height was small. (Sonic data are used here as they can be regarded as reliable for $1<k_{1} z<2$, as indicated in figure 2.) The results in figure 11 may seem to indicate that the spectra rise in level with increasing roughness, thus violating Townsend's Reynolds-number similarity hypothesis. An alternative explanation for this anomaly, however, may be that the results are a consequence of using the Taylor (1938) hypothesis in a flow with a large spread in convection velocities. This was investigated by Perry \& Li (1990) who found that the fractional spread in convection velocity, $\left(U_{1}-U\right) / U_{1}$, was considerably larger in rough-wall flows. Here, $U_{1}$ represents the free-stream velocity. They tested the effects 


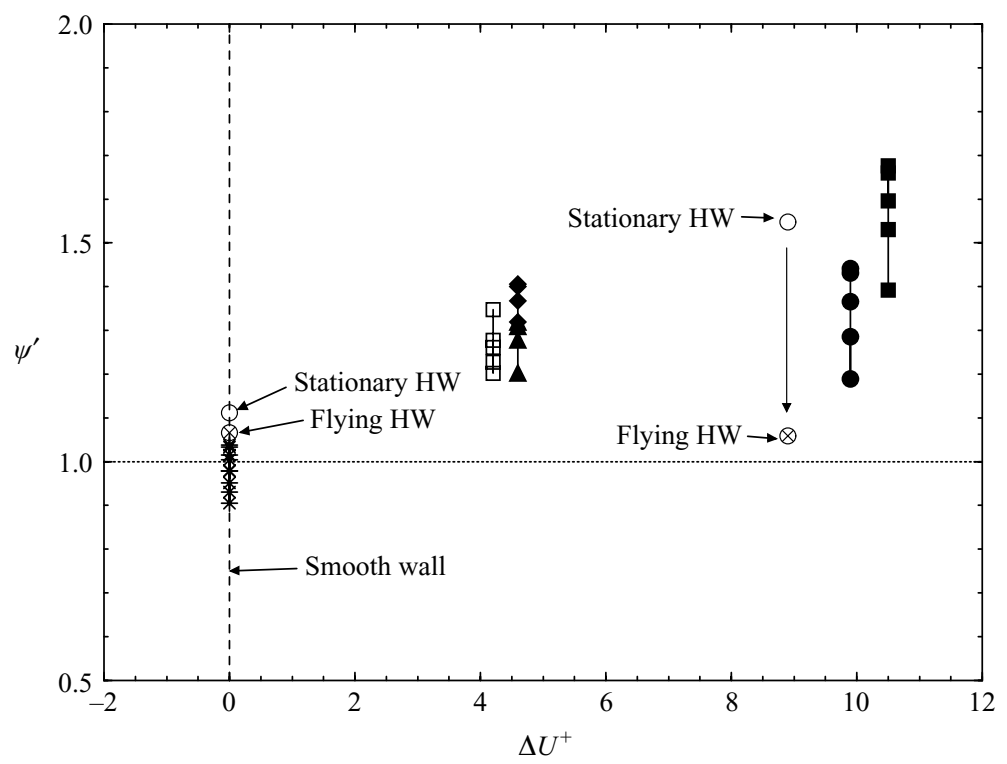

FIGURE 11. Integral parameter from equation (4.8) normalized with the corresponding value from equation (4.9) showing effects of spread in convection velocity on streamwise spectra. Solid symbols are atmospheric data as in figure 4. Open squares are from sonic anemometers at SLTEST facility in 2004. Stars are laboratory data corresponding to spectra in figure 10. Open circles and circles with crosses are Perry \& Li (1990) stationary and flying hot-wire results, respectively, for smooth and rough-wall flows.

of the spread in convection velocities on the spectra using a flying hot wire (their figure 18). The corresponding $\psi^{\prime}$ values from their data are shown in figure 11 . The rough-wall stationary-wire values from Perry \& Li (1990) agree well with the present atmospheric data and show a dramatic return to nominally smooth-wall values for the flying-wire case. They also made the same comparative measurements using a smoothwall (their figure 17) and found little effect (these data are also shown in figure 11). For wall-normal spectra on rough walls no discernible differences between flying and stationary measurements were observed (Perry \& Li 1990, figure 19), suggesting that the energy-containing motions for $\phi_{33}$ convect along with the mean velocity and are not influenced by the largest-scale motions in the flow (consistent with the attachededdy model). Therefore, it would seem that the atmospheric streamwise spectra may be biased by roughness-related convection velocity effects even up to $k_{1} z \sim 1$, but these effects would not be expected for the wall-normal spectra.

In summary, the streamwise spectra show results only qualitatively supporting the attached-eddy model. Strict quantitative issues cannot be resolved here given the poor convergence due to the very large time and length scales associated with the streamwise velocity component. Furthermore, the likely uncertainty due to the use of Taylor's hypothesis in flow with a large spread in convection velocities would also contaminate the spectra such that strict quantitative comparisons may not be valid.

\subsubsection{Wall-normal spectra}

The pre-multiplied wall-normal spectra from the atmosphere and laboratory, with outer- and inner-flow scaling, are shown in figures $12(a)$ and 12(b), respectively. Here, the spectra is much better converged for wavenumbers that contribute to wall-normal turbulence intensity, and convection velocity effects are expected to be 

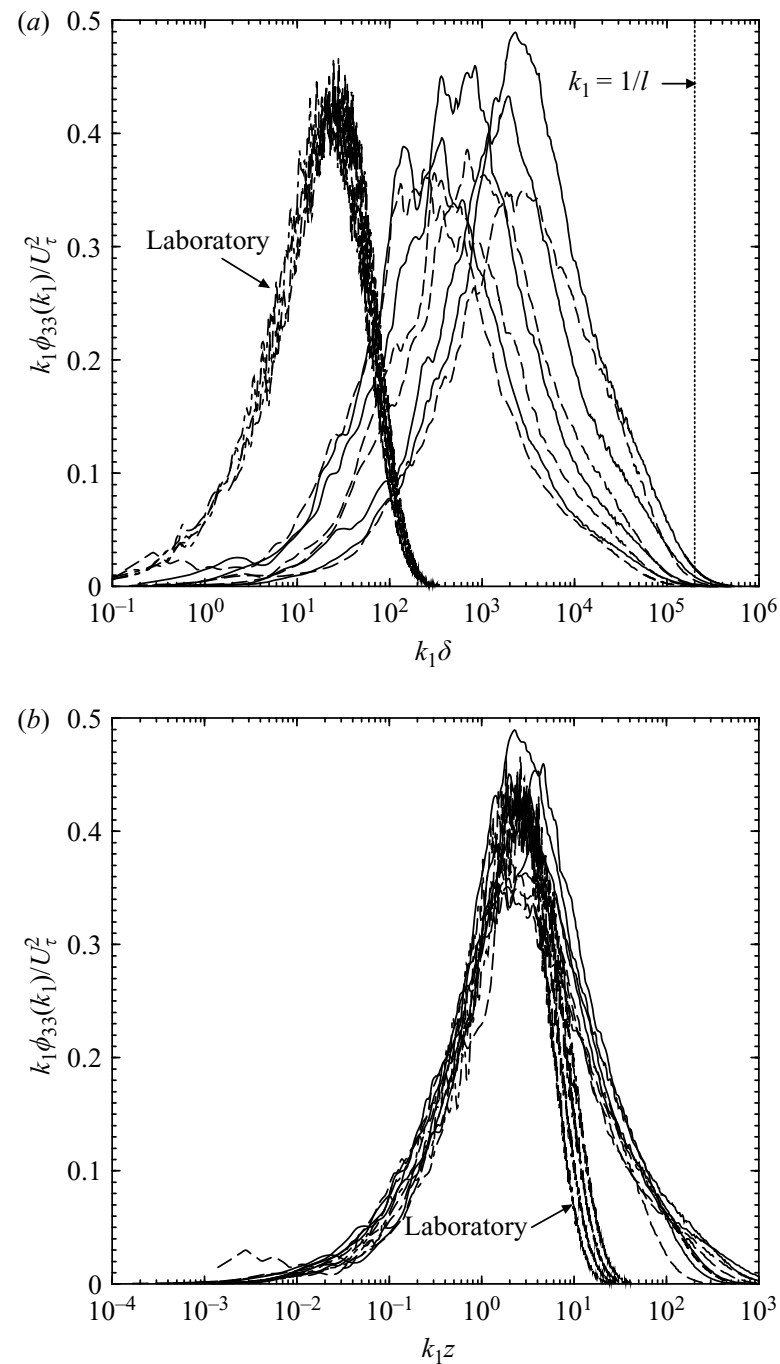

FIGURE 12. Wall-normal pre-multiplied spectra. Data as in figure 8. Also shown is the reciprocal of the hot-wire length $(l)$ for the atmospheric data. (a) Outer-flow scaling. (b) Innerflow scaling.

negligible (Perry \& Li 1990). According to the attached-eddy model, the wall-normal spectra with outer-flow scaling should have no region of similarity. With inner-flow scaling, the spectra are expected to collapse at low and moderate non-dimensional wavenumbers and in the $-5 / 3$ overlap region. At wavenumbers higher than the $-5 / 3$ region, the spectra should peel off with increasing $z^{+}$. Here, the spectra are seen to follow readily the similarity arguments of the attached-eddy model over three orders of magnitude in $\delta^{+}$. This marked similarity with inner-flow scaling, consistent with the attached-eddy model, suggests that the energy in the wall-normal component of velocity comes from eddies that scale with the local wall-normal position. This is different from the streamwise velocity fluctuations that have contributions from eddies larger than the local wall-normal height. 


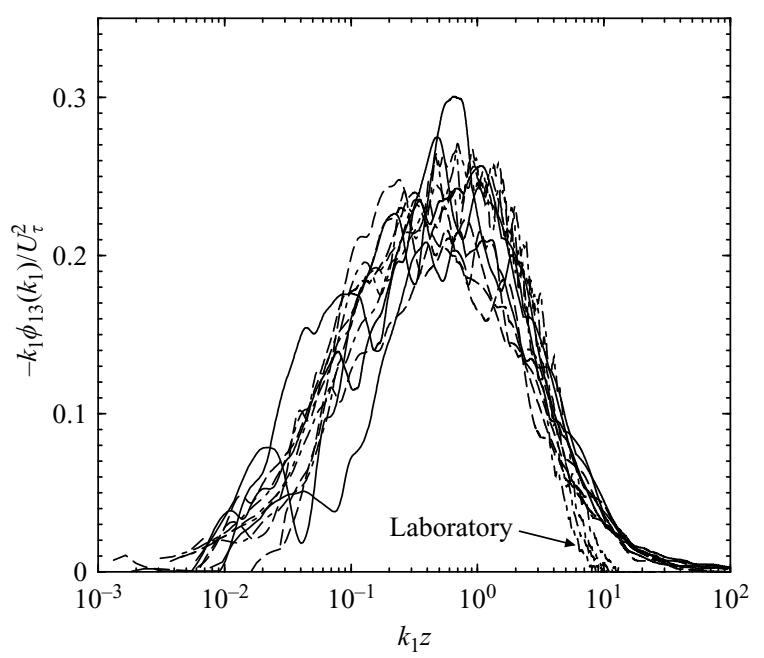

FIGURE 13. Pre-multiplied Reynolds-shear-stress spectra with inner-flow scaling. Data as in figure 9.

The wall-normal spectra are also consistent with the description of the ESL spectra given by Drobinski et al. (2004) and Hunt \& Morrison (2001), and the model given by Tchen (1953). Nikora (1999) suggests that the -1 region should be present in all the one-dimensional velocity spectra. The data seem to indicate that this is not the case. Note, however, Nikora's formulation is based on a simple phenomenological model that does not consider coherent structures, which are known to exist in turbulent boundary layers (i.e. Robinson 1991).

\subsubsection{Reynolds-shear-stress spectra}

The pre-multiplied cospectra with inner-flow scaling are shown in figure 13 . The cospectra, similar to the wall-normal spectra, are also found to be consistent with the suggested inner-flow scaling implied by the attached-eddy model. In figure 13, the cospectra appear to exhibit inner-flow scaling with similarity at moderate and low wavenumbers over all Reynolds numbers. Although there is some spread in the spectra at low wavenumbers, the spread is much lower than when scaled with Kolmogorov scales where no collapse is expected. With Kolmogorov scaling there is no similarity across the entire non-dimensional wavenumber range as expected (not shown). Similarly, there is no similarity with outer-flow scaling (not shown). All of these results agree with Townsend's attached-eddy hypothesis; similar to the wall-normal component of velocity, the major contributions to the Reynolds shear stress come from the local scales, i.e. the large 'inactive' scales do not contribute to the Reynolds shear stress.

Nikora (1999), Tchen (1953), and Jiménez (1999) all suggest that the Reynoldsshear-stress spectra should have a -1 region. However, as previously discussed, Nikora's model seems physically unrealistic, while the conclusion of Jiménez (1999) is based on data from Saddoughi \& Veeravalli (1994) which cannot be regarded as a canonical boundary layer. Their flow was over the acoustic tiles in the $80 \times 120 \mathrm{ft}^{2}$ NASA wind tunnel, and exhibit unusual behaviour for the wall-normal intensities. Furthermore, Tchen's model is given for turbulent shear flow without solid boundaries, and thus would not strictly be expected to wholly describe wall-bounded flow where the wall is assumed to limit the size of scales in the wall-normal direction. The results 
in figure 13 seem to indicate that there is no -1 region for the Reynolds-shear-stress spectra.

\section{Conclusions}

Data have been acquired in the surface layer of the atmospheric boundary layer at the SLTEST facility in the salt flats of western Utah. The site is unique in that it contains a long flat fetch with a relatively smooth surface (in comparison to typical atmospheric studies). Aside from the difficulties of resolving the large-scale low-frequency energy content and obtaining stationary winds, the use of the surface layer for near-wall high-Reynolds-number turbulence studies is promising. The meanflow data follow the classical fully rough-wall similarity formulation as discussed in Schlichting (1968) as well as the transitional rough-wall formulation developed by Ligrani \& Moffat (1986). Upon least-squares-error fitting the fully rough and transitionally rough formulations to the data, the calculated equivalent sand grain roughness heights are qualitatively similar to those physically observed on the salt flats $(\sim 10 \mathrm{~mm})$.

The streamwise and wall-normal turbulence intensity formulations, based on the attached-eddy model, appear to describe the data well over three orders of magnitude of $\delta^{+}$, from high atmospheric Reynolds numbers to lower laboratory Reynolds numbers. The formulations are founded on the physical arguments of the attached-eddy hypothesis and suggest the boundary-layer changes significantly with the Reynolds number, with an outer-flow influence felt all the way down to the wall. This influence, as shown by the extended streamwise formulation, increases with the Reynolds number. The good agreement with the similarity formulations suggests that the surface roughness $\left(k_{s}^{+} \approx 240, k_{s} / \delta \approx 8 \times 10^{-5}\right)$ does not affect the streamwise or the wall-normal turbulence intensities in the outer region of the flow, thus supporting Townsend's (1956) Reynolds-number similarity hypothesis for high Reynolds numbers. This is in disagreement with Krogstad \& Antonia (1999) (and others) who suggest Townsend's Reynolds-number similarity hypothesis is not valid above rough walls. The discrepancy appears to be resolved by requiring a high Reynolds number and a small roughness height relative to the large outer scales of the flow (i.e. the boundary-layer thickness), similar to the conclusions by Jiménez (2004). Studies that have not found outer-flow similarity have had small Reynolds numbers (and therefore an insufficient separation of inner- and outer-length scales), and/or large roughness heights relative to the outer-length scale.

The spectral scaling laws of the attached-eddy model (Perry et al. 1986; Perry \& Marusic 1995) are seen to work well for the wall-normal spectra and cospectra, with a clear lack of the 'inactive' (outer-flow) motion influence on the wall-normal velocity component. The wall-normal spectra appear to collapse at low and moderate wavenumbers when scaled with inner-flow variables. This collapse is consistent with laboratory data and shows inner-flow scaling over three orders of magnitude in the Reynolds number. The wall-normal spectra also have the classic $-5 / 3$ inertial subrange and do not have any large-scale energy contributions leading to a -1 region. The cospectra collapse reasonably well with inner-flow scaling at all wavenumbers, and agree with laboratory data. In agreement with the mean shear scaling arguments of Lumley (1967), the cospectra decay more rapidly in the inertial subrange $(-7 / 3$ slope $)$ than both the streamwise and wall-normal spectra ( $-5 / 3$ slope). No -1 slope spectral range is found in the cospectra. The good agreement between smooth-wall laboratory and rough-wall atmospheric wall-normal spectra and cospectra with inner-flow scaling 
also indicates strong support for Townsend's (1956) Reynolds-number similarity hypothesis and the attached-eddy model. Here, the energy-containing motions in the fully turbulent region of the flow are unaffected by roughness, except through its influence on $U_{\tau}$.

The streamwise spectra are seen to exhibit several trends consistent with the attached-eddy hypothesis, but when plotted in a pre-multiplied form, clear quantitative differences are noted between the low-Reynolds-number laboratory spectra and the atmospheric spectra. If these data are taken at face value, they indicate that the attached-eddy model does not complete the picture for the streamwise component. Certainly, this is possible as the attached-eddy model in its simplest form (Perry \& Marusic 1995) is restricted to a statistical description relying purely on an assemblage of self-similar eddies with varying population densities across a range of length scales. However, the streamwise spectra are shown to have considerable convergence and roughness-related convection velocity problems for low and moderate wavenumbers. The large time and length scales associated with the streamwise velocity component make converged measurements very difficult in the atmosphere. Comparisons with the flying hot-wire study of Perry \& Li (1990) indicate that the spread in the level of $\phi_{11}\left(k_{1} z\right) / U_{\tau}^{2}$ about $k_{1} z=1$ between the laboratory and atmospheric measurements can most probably be attributed to the use of Taylor's hypothesis in flow with a large spread in convection velocities. These measurement difficulties make it impossible for any firm conclusions to be made concerning the streamwise spectra at low and moderate wavenumbers. (These difficulties are much less important for the wallnormal spectra and the cospectra). For high wavenumbers, where the data can be regarded as reliable, the streamwise spectra have the classic $-5 / 3$ slope inertial subrange with scaling behaviour consistent with the overlap arguments of Perry et al. (1986).

The difficulties associated with the atmospheric study discussed here are distinctly different than those typically associated with well-controlled high-Reynolds-number laboratory facilities. This suggests a complementary boundary-layer study in a highReynolds-number laboratory flow, such as the High-Reynolds-Number Test Facility being developed at Princeton University, could prove to be fruitful.

The authors wish to gratefully acknowledge the support of the David and Lucile Packard Foundation. We also wish to thank Professor Joe Klewicki for hosting the SLTEST experiments, and Dr Nick Hutchins for assisting with some of the laboratory measurements.

\section{REFERENCES}

del Álamo, J. C., Jiménez, J., Zandonade, P. \& Moser, R. D. 2004 Scaling of the energy spectra of turbulent channels. J. Fluid Mech. 500, 135-144.

Bendat, J. S. \& Piersol, A. G. 2000 Random Data. John Wiley.

Bhaganagar, K., Kim, J. \& Coleman, G. 2004 Effect of roughness on wall-bounded turbulence. Flow Turb. Combust. 72, 463-492.

BruUn, H. H. 1995 Hot-Wire Anemometry. Oxford University Press.

Busch, N. E. 1973 The surface boundary layer. Boundary-Layer Met. 4, 213-240.

Coleman, H. W. \& Steele, W. G. 1989 Experimentation and Uncertainty Analysis for Engineers. John Wiley.

DeGraAfF, D. B. \& Eaton, J. K. 2000 Reynolds-number scaling of the flat-plate turbulent boundary layer. J. Fluid Mech. 422, 319-346.

Drobinski, P., Carlotti, P., Newsom, R. K., Banta, R. M., Foster, R. C. \& Redelsperger, J.-L. 2004 The structure of the near-neutral atmospheric surface layer. J. Atmos. Sci. 61, 699-714. 
Fernholz, H. H., Krause, E., Nockemann, M. \& Schober, M. 1995 Comparative measurements in the canonical boundary layer at $\operatorname{Re}_{\theta} \leqslant 6 \times 10^{4}$ on the wall of the German-Dutch windtunnel. Phys. Fluids 7, 1275-1281.

Flack, K. A., Schultz, M. P. \& Shapiro, T. A. 2005 Experimental support for Townsend's Reynolds number similarity hypothesis on rough walls. Phys. Fluids 17, 035102.

Foken, T. \& Skeib, G. 1983 Profile measurements in the atmospheric near-surface layer and the use of suitable universal functions for the determination of the turbulent energy exchange. Boundary-Layer Met. 25, 55-62.

GAD-El-HaK, M. \& BANDYOPADHYaY, P. R. 1994 Reynolds number effects in wall-bounded turbulent flows. Appl. Mech. Rev. 47, 307-365.

Gresko, L. W. 1988 Characteristics of wall pressure and near wall velocity in a flat plate turbulent boundary layer. Master's thesis, Massachusetts Institute of Technology.

HafEZ, S. H. M. 1991 The structure of accelerated turbulent boundary layers. PhD thesis, University of Melbourne, Australia.

Hama, F. R. 1954 Boundary-layer characteristics for smooth and rough surfaces. Trans. Soc. Nav. Arch. Marine Engng 62, 333-358.

Hogstrom, U. 1988 Non-dimensional wind and temperature profiles in the atmospheric surface layer: a re-evaluation. Boundary-Layer Met. 42, 55-78.

Hogstrom, U., Hunt, J. C. R. \& Smedman, A. 2002 Theory and measurements for turbulence spectra and variances in the atmospheric neutral surface layer. Boundary-Layer Met. 103, $101-124$.

Hommema, S. E. \& Adrian, R. J. 2003 Packet structures of surface eddies in the atmospheric boundary layer. Boundary-Layer Met. 106, 147-170.

Hunt, J. C. R. \& CARLotTi, P. 2001 Statistical structure at the wall of the high Reynolds number turbulent boundary layer. Flow Turb. Combust. 66, 453-475.

Hunt, J. C. R. \& Morrison, J. F. 2001 Eddy structure in turbulent boundary layers. Eur. J. Mech. B Fluids 19, 673-694.

JimÉneZ, J. 1999 The physics of wall turbulence. Physica A 263, 252-262.

JimÉnez, J. 2004 Turbulent flows over rough walls. Annu. Rev. Fluid Mech. 36, 173-96.

JimÉnez, J. \& Pinelli, A. 1999 The autonomous cycle of near-wall turbulence. J. Fluid Mech. 389, $335-359$.

Jones, M. B., Marusic, I. \& Perry, A. E. 2001 Evolution and structure of sink-flow turbulent boundary layers. J. Fluid Mech. 428, 1-27.

Kaimal, J. C. \& Finnigan, J. J. 1994 Atmospheric Boundary Layer Flows. Oxford University Press.

Kaimal, J. C., Wyngaard, J. C., Izumi, Y. \& Cote, O. R. 1972 Spectral characteristics of surfacelayer turbulence. Q. J. R. Met. Soc. 98, 563-589.

Klewicki, J. C., Foss, J. F. \& Wallace, J. M. 1998 High Reynolds number $\left[R_{\theta}=\mathrm{O}\left(10^{6}\right)\right]$ boundary layer turbulence in the atmospheric surface layer above Western Utah's salt flats. In Flow at Ultra-High Reynolds and Rayleigh Numbers (ed. R. J. Donnelly \& K. R. Sreenivasan), pp. 450-466. Springer.

Krogstad, P. \& Antonia, R. A. 1999 Surface roughness effects in turbulent boundary layers. Exps. Fluids 27, 450-460.

Kunkel, G. J. 2003 An experimental study of the high Reynolds number turbulent boundary layer. $\mathrm{PhD}$ thesis, The University of Minnesota.

LEwKowicz, A. K. 1982 An improved universal wake function for turbulent boundary layers and some of its consequences. Z. Flugwiss. Weltraumforschung 6, 261-266.

Ligrani, P. M. \& Moffat, R. J. 1986 Structure of transitionally rough and fully rough turbulent boundary layers. J. Fluid Mech. 162, 69-98.

Lumley, J. L. 1967 Similarity and the turbulent energy spectrum. Phys. Fluids 10, 855-858.

Lumley, J. L. \& Panofsky, H. A. 1964 The Structure of Atmospheric Turbulence. John Wiley.

Marusic, I. \& Kunkel, G. J. 2003 Streamwise turbulence intensity formulation for flat-plate boundary layers. Phys. Fluids 15, 2461-2464.

Marusic, I., Kunkel, G. J. \& Porte-Agel, F. 2001 Experimental study of wall boundary conditions for large-eddy simulation. J. Fluid Mech. 446, 309-320.

Marusic, I., Kunkel, G. J., ZhaO, R. \& SMits, A. J. 2004 Turbulence intensity similarity formulations for wall-bounded flows. In Advances in Turbulence X: Proceedings of the Tenth European Turbulence Conference. 
Marusic, I. \& Perry, A. E. 1995 A wall-wake model for the turbulence structure of boundary layers. Part 2. Further experimental support. J. Fluid Mech. 298, 389-407.

Marusic, I., Uddin, A. K. M. \& Perry, A. E. 1997 Similarity law for the streamwise turbulence intensity in zero-pressure-gradient turbulent boundary layers. Phys. Fluids 9, 3718-3726.

MetzGer, M. M. 2002 Scalar dispersion in high Reynolds number turbulent boundary layers. PhD thesis, The University of Utah.

Metzger, M. M. \& KLewicki, J. C. 2001 A comparative study of near-wall turbulence in high and low Reynolds number boundary layers. Phys. Fluids 13, 692-701.

Monin, A. S. \& Yaglom, A. M. 1971 Statistical Fluid Mechanics: Mechanics of Turbulence, vol. 1, The MIT Press.

Morrison, J. F., McKeon, B. J., Jiang, W. \& Smits, A. J. 2004 Scaling of the streamwise velocity component in turbulent pipe flow. J. Fluid Mech. 508, 99-131.

Nickels, T. B., Hafez, S., Marusic, I. \& Chong, M. S. 2004 Evidence of the -1-law in a high Reynolds number turbulent boundary layer. In Proc. 15th Australasian Fluid Mech. Conf. paper AFMC00118. University of Sydney, Sydney, Australia.

NikorA, V. 1999 Origin of the '-1' spectral law in wall-bounded turbulence. Phys. Rev. Lett. 83, 734-736.

Pahlow, M., Parlange, M. B. \& Porte-Agel, F. 2001 On Monin-Obukhov similarity in the stable atmospheric boundary layer. Boundary-Layer Met. 99, 225-248.

Perry, A. E. \& Abell, C. J. 1975 Scaling laws for pipe-flow turbulence. J. Fluid Mech. 67, 257-271.

Perry, A. E., Henbest, S. M. \& Chong, M. S. 1986 A theoretical and experimental study of wall turbulence. J. Fluid Mech. 165, 163-199.

Perry, A. E. \& LI, J. D. 1990 Experimental support for the attached eddy hypothesis in zeropressure-gradient turbulent boundary layers. J. Fluid Mech. 218, 405-438.

Perry, A. E. \& Marusic, I. 1995 A wall-wake model for the turbulence structure of boundary layers. Part 1. Extension of the attached eddy hypothesis. J. Fluid Mech. 298, 361-388.

Pope, S. B. 2000 Turbulent Flows. Cambridge University Press.

Priyadarshana, P. J. A. \& Klewicki, J. C. 2004 Study of the motions contributing to the Reynolds stress in high and low Reynolds number turbulent boundary layers. Phys. Fluids 16, 45864600.

Raupach, M. R., Antonia, R. A. \& Rajagopalan, S. 1991 Rough-wall turbulent boundary layers. Appl. Mech. Rev. 44, 1-25.

Robinson, S. K. 1991 Coherent motion in the turbulent boundary layer. Annu. Rev. Fluid Mech. 23, 601-639.

Saddoughi, S. G. \& Veeravalli, S. V. 1994 Local isotropy in turbulent boundary layers at high Reynolds number. J. Fluid Mech. 268, 333-372.

Schlichting, H. 1968 Boundary-Layer Theory, 6th edn. McGraw-Hill.

SPALART, P. R. 1988 Direct simulation of a turbulent boundary layer up to $R_{\theta}=1410$. J. Fluid Mech. 187, 61-98.

StUll, R. B. 1988 An Introduction to Boundary Layer Meteorology. Kluwer.

TAYLOR, G. I. 1938 The spectrum of turbulence. Proc. R. Soc. Lond. A 164, 476-490.

TChen, C. M. 1953 On the spectrum of energy in turbulent shear flow. J. Res. Natl Bureau Stand. 50, 51-62.

Tennekes, H. \& Lumley, J. L. 1972 A First Course in Turbulence, vol. 2. The MIT Press.

Townsend, A. A. 1956 The Structure of Turbulent Shear Flow. Cambridge University Press.

Townsend, A. A. 1976 The Structure of Turbulent Shear Flow, 2nd edn., Cambridge University Press.

Waleffe, F. 1997 On a self-sustaining process in shear flows. Phys. Fluids 9, 883-900.

WeBER, R. O. 1999 Remarks on the definition and estimation of friction velocity. Boundary-Layer Met. 93, 197-209.

WyngaArd, J. C. 1992 Atmospheric turbulence. Annu. Rev. Fluid Mech. 24, 205-233.

WyngaARD, J. C. \& Cote, O. R. 1972 Cospectral similarity in the atmospheric surface layer. $Q . J . R$. Met. Soc. 98, 590-603.

YAVUZKURT, S. 1984 A guide to uncertainty analysis of hot-wire data. Trans. ASME I: J. Fluids Engng 106, 181-186. 


\section{University Library}

\section{- M M N E R VA A gateway to Melbourne's research publications}

Minerva Access is the Institutional Repository of The University of Melbourne

Author/s:

Kunkel, GJ;Marusic, I

Title:

Study of the near-wall-turbulent region of the high-Reynolds-number boundary layer using an atmospheric flow

Date:

2006-02-10

Citation:

Kunkel, G. J. \& Marusic, I. (2006). Study of the near-wall-turbulent region of the highReynolds-number boundary layer using an atmospheric flow. JOURNAL OF FLUID MECHANICS, 548 (-1), pp.375-402. https://doi.org/10.1017/S0022112005007780.

Publication Status:

Published

Persistent Link:

http://hdl.handle.net/11343/34788 